Notes and Comments

\title{
Chemical compositions of amphiboles in hematite-bearing schists from the Saruta-gawa area in the Sanbagawa belt, central Shikoku, Japan
}

\author{
Yasuyuki BANNO ${ }^{1}$
}

\begin{abstract}
Yasuyuki BANNo (2001) Chemical compositions of amphiboles in hematite-bearing schists from the Saruta-gawa area in the Sanbagawa belt, central Shikoku, Japan. Bull. Geol. Surv. Japan, vol. 52 (9), p. 425-443, 3 figs, 10 tables.
\end{abstract}

\begin{abstract}
Amphibole and other minerals in the hematite-bearing basic and quartz schists from the Saruta-gawa area in the Sanbagawa belt, central Shikoku were analyzed by the electron microprobe. The data presented comprise 221 analyses of amphibole, and 90 analyses of epidote, chlorite, garnet, muscovite, albite, hematite, magnetite and stilpnomelane. All the minerals in this study occur from the hematite-bearing schists in the albite-biotite zone of high metamorphic grade. These chemical data were used in order to discuss the Sanbagawa retrograde metamorphism in the Saruta-gawa area (Banno, 2000).
\end{abstract}

\section{Introduction}

The Sanbagawa belt is a regional coherent high$\mathrm{P} / \mathrm{T}$ metamorphic belt that extends from east to west for about $800 \mathrm{~km}$ in the Southwest Japan. The peak metamorphic condition has been estimated in many previous studies, and characteristics of the metamorphic field gradient were compiled by Banno and Sakai (1989) and Enami et al. (1994).

The chemistry of amphibole in hematite-bearing basic and quartz schists of the Sanbagawa belt can be used as a sensitive indicator of metamorphic grade (Otsuki and Banno, 1990; Enami et al., 1994). Otsuki and Banno (1990) studied the compositional zonation of amphibole crystals in the hematite-bearing basic schists in the Asemi-gawa area, central Shikoku, and showed that the zoned amphibole exhibited decreasing $\mathrm{Al}_{2} \mathrm{O}_{3}$ contents towards the crystal margin and was usually rimmed with actinolite. They proposed the phase relation of the amphiboles associated with chlorite, epidote, muscovite, albite, quartz and hematite, and considered that the zonal structure indicates the rapid pressure release during the early stage of retrograde metamorphism. The amphibole in the hematite-bearing basic schists from the Saruta-gawa area located in $8 \mathrm{~km}$ north of the Asemi-gawa area (Fig. 1), however, has usually a rim of retrograde sodic

${ }^{1}$ Institute of Geoscience, GSJ amphibole instead of actinolite (Hara et al., 1990), and Banno (2000) showed that the Saruta-gawa samples experienced retrograde metamorphism under higher $\mathrm{P} / \mathrm{T}$ condition than the Asemi-gawa samples. Thus, the compositional trend of zoned amphiboles in hematite-bearing schists is important for understanding retrograde metamorphism, although, in the literature, few analytical data on amphibole chemistry were given due to editorial limitations. Therefore, in this paper, (1) all the chemical data of the Saruta-gawa amphiboles and (2) representative analyses of minerals associated with the amphibole, which were studied in Banno (2000), are presented.

\section{Sample numbers}

Each sample has two sample numbers as shown in Table 1. One is the original number (e.g., YB108), which was presented in Banno (2000), and the other is registration number of the petrological collection of the Geological Museum, AIST, Tsukuba (e.g., GSJ R76512). In this paper, only the original numbers are given to simplify the description.

\section{Abbreviations in the text}

Abbreviations used in the text are as follows: Keywords: chemical composition, amphibole, basic schist, quartz schist, Sanbagawa belt, Saruta-gawa area 


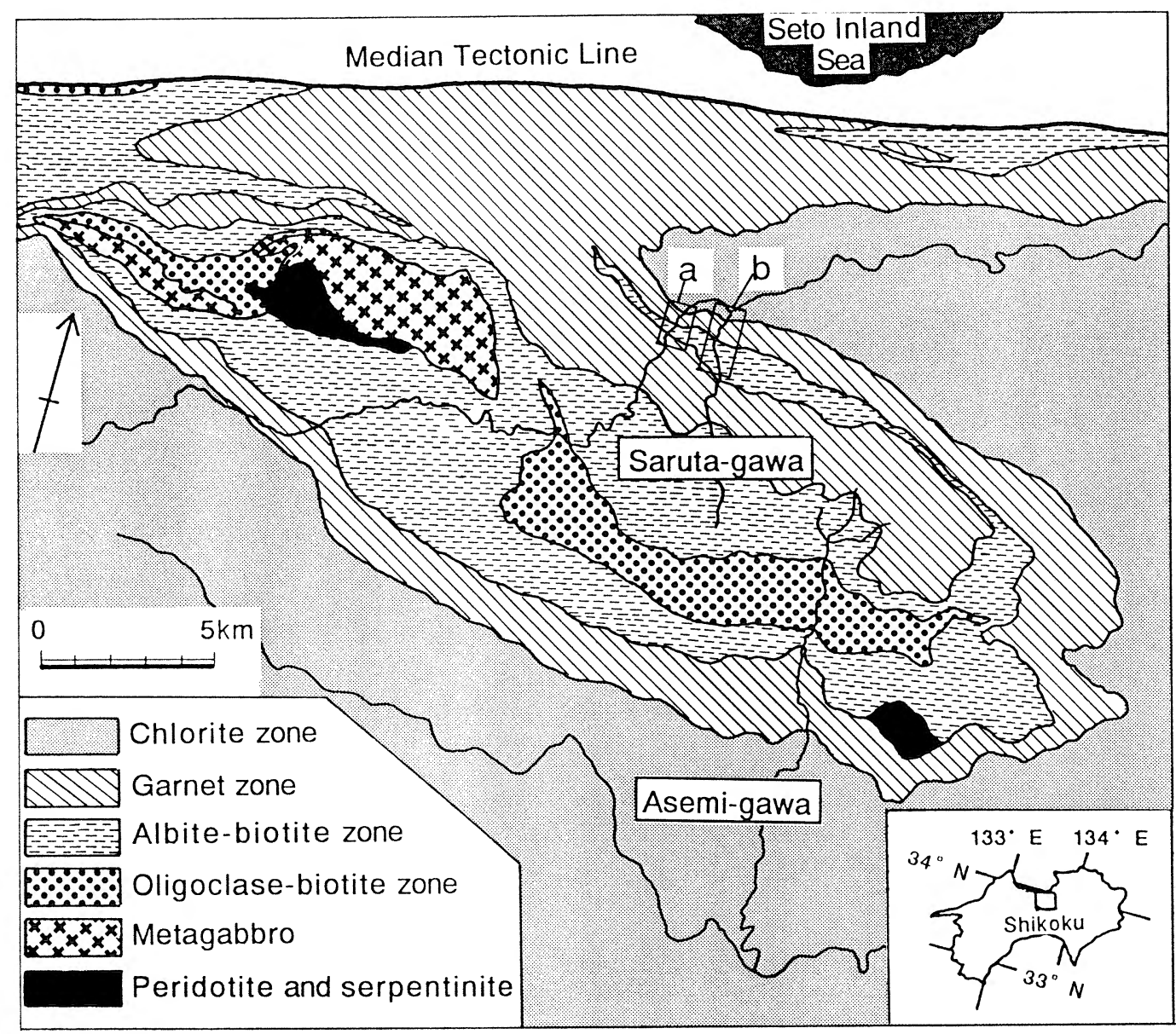

Fig. 1 Metamorphic zones in the Sanbagawa metamorphic belt, central Shikoku (simplified from Higashino, 1990). Small rectangles marked with $\mathrm{a}$ and $\mathrm{b}$ indicate the locations of traverse maps shown in Fig. 2a and b, respectively.

${ }^{[4]} \mathrm{Al}=$ tetrahedral aluminum, ${ }^{[\mathrm{A}]}(\mathrm{Na}+\mathrm{K})=$ sodium and potassium in A sites, ${ }^{[\mathrm{B}]} \mathrm{Na}=$ sodium in $\mathrm{M} 4$ sites, ${ }^{[6]} \mathrm{Ti}=$ octahedral titanium.

\section{Outline of geology and mineral assemblages}

The Saruta-gawa area is located in Iyo-Mishima city, Ehime Prefecture in central Shikoku, and a part of the Sazare area of Higashino et al. (1984). Traverse maps and sample locations of the Saruta-gawa area are shown in Fig. 2. The Sanbagawa schists generally trend $\mathrm{E}-\mathrm{W}$ and dip to the north. However, in the Saruta-gawa area, local southward dips caused by later folding are observed. The bed of this area strikes roughly WNW and dips about $40^{\circ}$ to $90^{\circ}$ south. On the basis of the mineral assemblages in pelitic schists, this area is divided into three mineral zones, the chlorite, the garnet and the albite-biotite zones in ascending order of metamorphic grade (e.g., Higashino, 1990). The analyzed samples were four basic schists and four quartz schists and were all collected from the albitebiotite zone (Fig. 2). Mineral assemblages of the sam- ples are shown in Table 1. All the samples contain chlorite, epidote, muscovite, albite, quartz and hematite. Garnet was found exclusively in quartz schist.

\section{Outline of mineral chemistry}

The chemical characteristics of amphibole and other minerals listed in Tables 2 to 10 are briefly described in this chapter. Minerals in eight samples were examined by a JEOL JXA-8800R electron-probe microanalyzer at AIST. Accelerating voltage, specimen current and beam diameter were kept at $15 \mathrm{kV}$, $12 \mathrm{nA}$ on a Faraday cup and $3 \mu \mathrm{m}$, respectively. More detailed descriptions were given in Banno (2000).

\subsection{Amphibole}

All the analyses of amphiboles are listed in Table 2. The $\mathrm{Fe}^{3+} / \mathrm{Fe}^{2+}$ of amphibole is calculated for assuming 13 total cations exclusive of $\mathrm{K}, \mathrm{Na}$ and $\mathrm{Ca}$ on the basis of $23 \mathrm{O}$ atoms. The nomenclature of amphibole conforms to Leake et al. (1997). Some sodic-calcic amphiboles with ${ }^{[4]} \mathrm{Al}$ contents higher than 0.5 per for- 
(a)
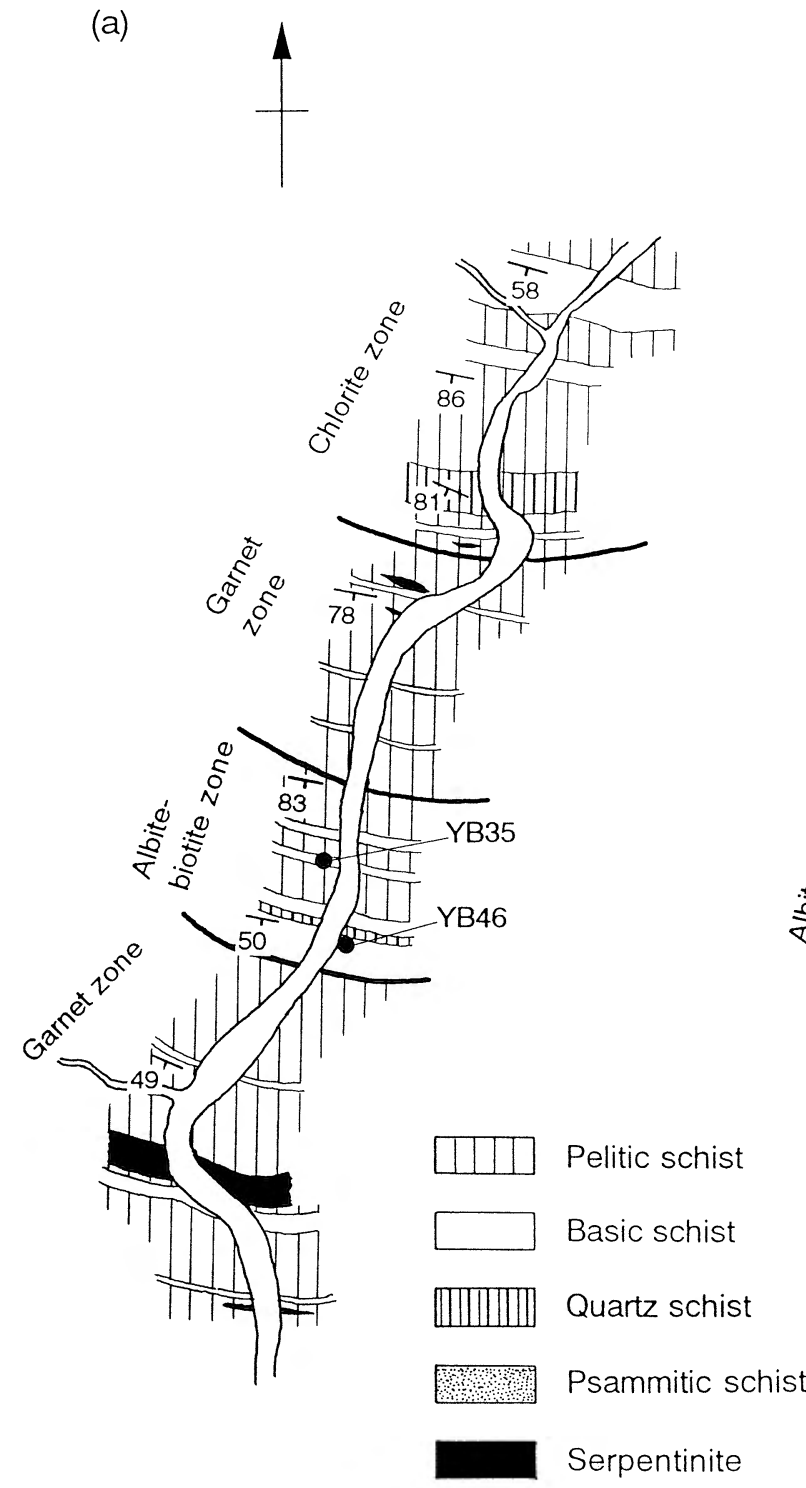

(b)

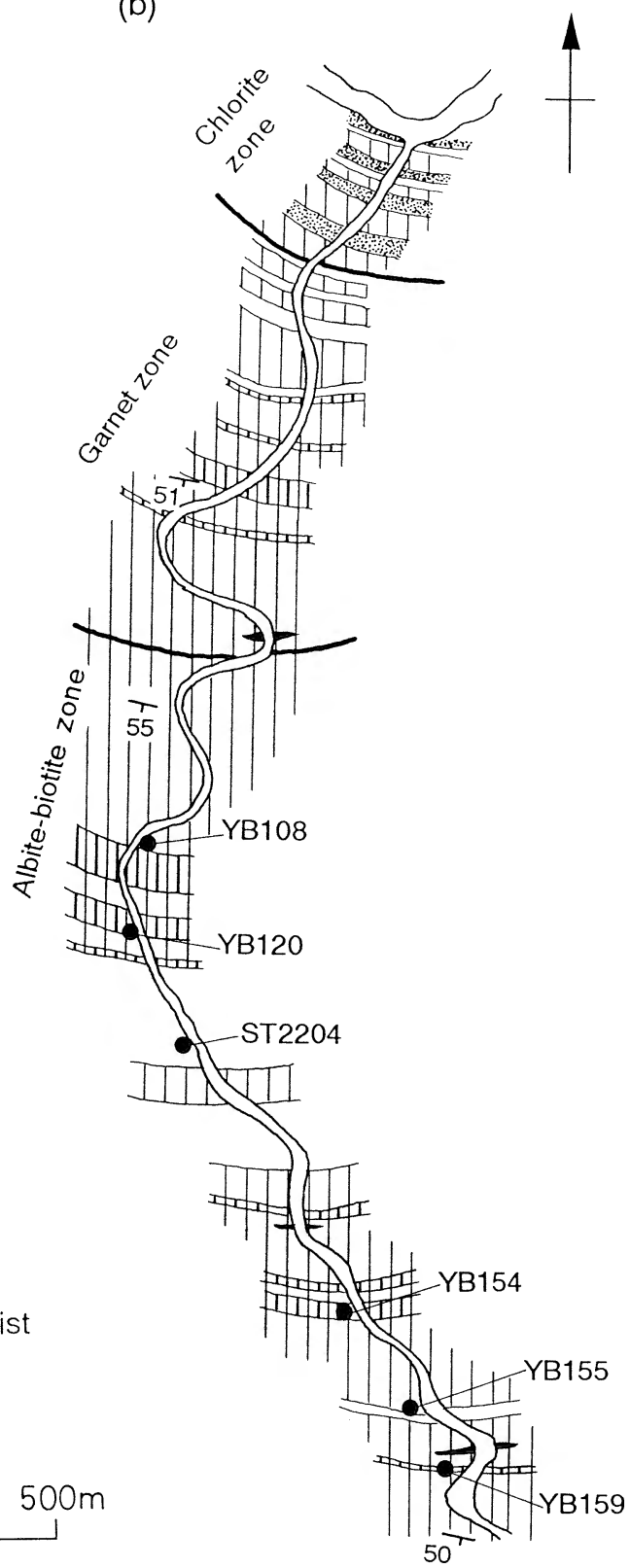

Fig. 2 Traverse maps and sample locations of the Saruta-gawa area.

mula unit (pfu) show ${ }^{[\mathrm{A}]}(\mathrm{Na}+\mathrm{K})>0.5 \mathrm{pfu}$, and these are magnesiokatophorites. All the sodic-calcic amphiboles containing ${ }^{[4]} \mathrm{Al}>0.5$ pfu are classified as barroisite in this paper to simplify the mineral description.

Amphibole occurs as matrix minerals and inclusions in the albite porphyroblasts. In this study, only matrix amphiboles were analyzed. The amphiboles possess Al-rich core and Al-poor mantle. Zoned crystals of amphiboles were examined by line scanning profiles from Al-rich core to Al-poor mantle. In Table 2 , chemical data obtained from different spots in the same amphibole crystal are arranged in a sequence from core to rim. The Al-rich core has a barroisitic composition. In the mantle part, ${ }^{[\mathrm{B}]} \mathrm{Na}$ increases with decreasing ${ }^{[4]} \mathrm{Al}$ towards margin, which has a winchit- e-magnesioriebeckite composition [cf. Fig. 3a and b in Banno (2000)]. The barroisite-winchite-magnesioriebeckite composite crystal is sometimes rimmed with actinolite and/or winchite with low ${ }^{[\mathrm{B}]} \mathrm{Na}$ and ${ }^{[4]} \mathrm{Al}$. In the mantle part of this zoning pattern, ${ }^{[\mathrm{B}]} \mathrm{Na}$ increases with decreasing ${ }^{[4]} \mathrm{Al}$, then decreases towards the margin [cf. Fig. 3c-e in Banno (2000)].

Compositional ranges of Al-rich cores and Al-poor mantles are shown on an ${ }^{[4]} \mathrm{Al}-{ }^{[\mathrm{B}]} \mathrm{Na}$ diagram (Fig. 3). The barroisite core in basic schists is generally poorer in ${ }^{[\mathrm{B}]} \mathrm{Na}$ than in quartz schists. The ${ }^{[\mathrm{B}]} \mathrm{Na}$ content of the mantle in basic schists extends to lower values than in quartz schists. The systematic difference in ${ }^{[\mathrm{B}]} \mathrm{Na}$ between basic and quartz schists can be explained by differences in oxygen fugacity between the two rock 


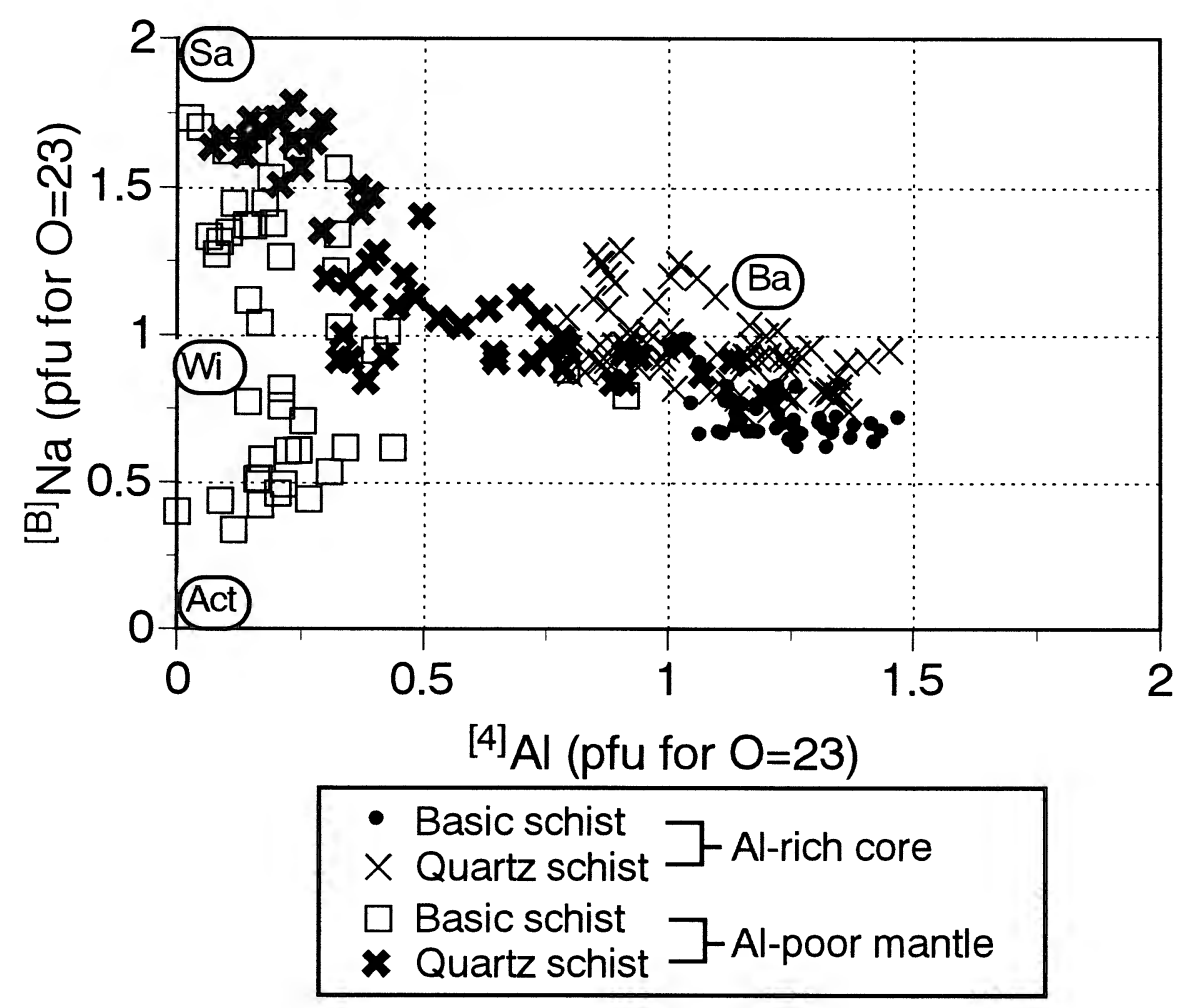

Fig. 3 Compositional ranges of Al-rich core and Al-poor mantle in basic and quartz schists from the Saruta-gawa area. $\mathrm{Ba}=$ barroisite, $\mathrm{Sa}=$ sodic amphibole, $\mathrm{Wi}=$ winchite, Act $=$ actinolite.

types (Banno, 2000).

\subsection{Minerals other than amphibole}

Representative analyses of epidote, chlorite, garnet, muscovite, albite, hematite, magnetite and stilpnomelane are tabulated in Tables 3-10, respectively. Epidote is commonly zoned with decreasing ferrian component $\left[\mathrm{Y}_{\mathrm{Fe}^{3+}}=\mathrm{Fe}^{3+} /\left(\mathrm{Fe}^{3+}+\mathrm{Al}\right)\right]$ from core to rim (e.g., ST2204, YB35), although the zoning with reverse sense is observed in YB108. The ranges of $\mathrm{YFe}_{\mathrm{Fe}^{3+}}$ of epidotes in basic and quartz schists are $0.21-0.31$ and $0.27-0.33$, respectively. Total iron was assumed to be $\mathrm{Fe}_{2} \mathrm{O}_{3}$.

Compositional ranges of $\mathrm{X}_{\mathrm{Sps}}$ (=spessartine component) in garnet are generally 0.19-0.72, although garnet in YB154 shows lower $\mathrm{X}_{\mathrm{Sps}}(0.13-0.20)$. Except for YB154, garnet occurs as zoned crystals in which $\mathrm{Mn}$ contents decrease from core $\left(\mathrm{X}_{\mathrm{Sps}}=0.55-0.72\right)$ to $\operatorname{rim}\left(\mathrm{X}_{\mathrm{Sps}}=0.19-0.43\right) . \quad \mathrm{X}_{\mathrm{Grs}}$ (= grossular component) in garnet from YB159 increases outwards, and attains its maximum of 0.20 at an intermediate position between core and rim, and then decreases towards the outermost rim. The chemical data showing the highest $\mathrm{X}_{\mathrm{Grs}}$ contents are marked with "inter" in Table $5 . \mathrm{Mn}$ in garnet from YB154 decreases from the core $\left(\mathrm{X}_{\mathrm{Sps}}=0.20\right)$ towards the rim $\left(\mathrm{X}_{\mathrm{Sps}}=0.13\right)$. The ferric iron contents of garnet were calculated on the basis of $\left(\mathrm{A} 1+\mathrm{Fe}^{3+}\right)$ : $\left(\mathrm{Fe}^{2+}+\mathrm{Mn}+\mathrm{Mg}+\mathrm{Ca}\right)=2: 3$.

Muscovite is phengitic with a Si content of 3.20-3.35 pfu $(\mathrm{O}=11)$. The $\mathrm{Na} /(\mathrm{Na}+\mathrm{K})$ ratio is $0.05-0.15$. Fer- rous iron content was estimated as $\mathrm{Si}+\mathrm{Ti}-3=\mathrm{Fe}^{2+}+$ $\mathrm{Mg}+\mathrm{Mn}(\mathrm{O}=11)$, assuming $\left(\mathrm{Fe}^{2+}, \mathrm{Mg}, \mathrm{Mn}\right) \mathrm{SiAl}_{-1} \mathrm{Al}_{-1}$ and ${ }^{[4]} \mathrm{Al}^{[6]} \mathrm{TiSi}_{-1} \mathrm{Al}_{-1}$ substitutions.

Hematite includes fine exsolution lamellae of ilmenite. Ti contents of the host hematite are less than $0.19 \mathrm{pfu}$. In ST2204, hematite including the ilmenite lamellae is rimmed with lamellae-free hematite. The former has higher Ti contents $(0.15-0.19 \mathrm{pfu})$ than the latter $(0.01-0.10 \mathrm{pfu}) . \mathrm{Fe}^{3+} / \mathrm{Fe}^{2+}$ ratio was calculated assuming total cation $=2(\mathrm{O}=3)$.

Magnetite occurs in YB108 and coexists with hematite. It contains small amounts of $\mathrm{SiO}_{2}(0.6-0.7 \mathrm{wt} . \%)$. $\mathrm{Fe}^{3+} / \mathrm{Fe}^{2+}$ ratio was calculated on the assumption of total cation $=3$ for $\mathrm{O}=4$.

Stilpnomelane occurs in YB35, and is relatively rich in $\mathrm{Mn}(0.18-0.20 \mathrm{pfu})$. Formulae of stilpnomelane were calculated assuming $\mathrm{Si}=8$ with total iron as $\mathrm{Fe}^{2+}$.

Albite shows low $\mathrm{Ca} /(\mathrm{Ca}+\mathrm{Na})(<0.02) . \mathrm{Mg} /(\mathrm{Mg}+$ $\mathrm{Fe}^{2+}$ ) values of chlorite ranges from $0.45-0.69$.

Acknowledgements: The author is indebted to M. Haruna for critical reading the manuscript and to M. Enami for providing the sample ST2204. Thanks are due to $\mathrm{H}$. Hirano for his improvements of the manuscript. 


\section{References}

Banno, S. and Sakai, C. (1989) Geology and metamorphic evolution of the Sanbagawa metamorphic belt, Japan. In: Daly, J.S., Cliff, R. A., Yardley, B. W.D. eds., Evolution of Metamorphic Belts. Geol. Soc. London, Spec. Pub., no. 43, 519-532.

Banno, Y. (2000) Intermediate high-pressure exhumation of the northern segment of the Sanbagawa belt, Saruta-gawa area, central Shikoku, Japan. Lithos, 50, 289-303.

Enami, M., Wallis, S. R. and Banno, Y. (1994) Paragenesis of sodic pyroxene-bearing quartz schists: implications for P-T history of the Sanbagawa belt. Contrib. Mineral. Petrol., 116, 182-198.

Hara, I., Shiota, T., Hide, K., Okamoto, K., Takeda, K., Hayasaka, Y. and Sakurai, Y. (1990) Nappe structure of the Sambagawa belt. J. Metamorph. Geol., 8, 441-456.

Higashino, T. (1990) Metamorphic zones of the Sambagawa metamorphic belt in central Shikoku, Japan. Jour. Geol. Soc. Japan, 96, 703718. (in Japanese with English abstract)
Higashino, T., Sakai, C., Kurata, H., Enami, M., Hosotani, H., Enami, Makiko and Banno, S. (1984) Electron microprobe analyses of rockforming minerals from the Sanbagawa metamorphic rocks, Shikoku Part II. Sazare, Kotu and Bessi areas. Sci. Rep. Kanazawa Univ., 29, 37-64.

Leake, B.E., Woolley, A.R., Arps, C.E.S., Birch, W. D., Gilbert, M.C., Grice, J.D., Hawthorne, F.C., Kato, A., Kisch, H. J., Krivovichev, V. G., Linthout, K., Laird, J., Mandarino, J., Maresch, W. V., Nickel, E.H., Rock, N.M.S., Schumacher, J.C., Smith, D. C., Stephenson, N.C. N., Ungaretti, L., Whittaker, E. J.W. and Youzhi, G. (1997) Nomenclature of amphiboles: Report of the Subcommittee on Amphiboles of the International Mineralogical Association, Commission on New Minerals and Mineral Names. Eur. J. Mineral., 9, 623-651.

Otsuki, M. and Banno, S. (1990) Prograde and retrograde metamorphism of hematite-bearing basic schists in the Sanbagawa belt in central Shikoku. J. Metamorph. Geol., 8, 425-439.

Received September 28, 2001

Accepted October 30, 2001

Abbreviations used in Tables $1-10$ are as follows.

(Tables $1-10) \quad B S=$ basic schist, $Q S=$ quartz schist.

(Tables 2 and 4$){ }^{[4]} \mathrm{Al}=$ tetrahedral aluminum, ${ }^{[6]} \mathrm{Al}=$ octahedral aluminum.

(Table 1) Grt=garnet, Amp=amphibole, $\mathrm{Ep}=$ epidote, $\mathrm{Chl}=\mathrm{chlorite}, \mathrm{Ms}=$ muscovite, Car $=\mathrm{carbonate}, \mathrm{Ttn}=\mathrm{titanite}, \mathrm{Rt}=\mathrm{rutile}$, $\mathrm{Ab}=$ albite, $\mathrm{Qtz}=$ quartz, Hem = hematite, Ap=apatite, Mag= magnetite, Tur=tourmaline, Ilm=ilmenite, Stp=stilpnomelane.

(Table 2) $\mathrm{Ba}=$ barroisite, $\mathrm{Wi}=$ winchite, $\mathrm{Mrb}=$ magnesioriebeckite, $\mathrm{Gln}=$ glaucophane, Act $=$ actinolite, ${ }^{[\mathrm{B}]} \mathrm{Na}=\mathrm{sodium}$ in $\mathrm{M} 4$ sites, ${ }^{[\mathrm{A}]} \mathrm{Na}=$ sodium in $\mathrm{A}$ sites, $\mathrm{X}_{\mathrm{Mg}}=\mathrm{Mg} /\left(\mathrm{Mg}+\mathrm{Fe}^{2+}\right), \mathrm{X}_{\mathrm{Fe}^{3+}}=\mathrm{Fe}^{3+} /\left(\mathrm{Fe}^{3+}+{ }^{[6]} \mathrm{Al}\right)$, * total iron as $\mathrm{FeO}$, \# ${ }^{*}=$ the highest ${ }^{[\mathrm{B}]} \mathrm{Na}$ position in the mantle, n.d. $=$ not determined.

(Table 3) $\mathrm{Y}_{\mathrm{Fe}^{3+}}=\mathrm{Fe}^{3+} /\left(\mathrm{Fe}^{3+}+\mathrm{Al}\right),{ }^{*}=$ total iron as $\mathrm{Fe}_{2} \mathrm{O}_{3}$.

(Table 4) $\quad \mathrm{X}_{\mathrm{Mg}}=\mathrm{Mg} /\left(\mathrm{Mg}+\mathrm{Fe}^{2+}\right)$.

(Table 5) inter=intermediate position, showing the highest grossular component, between core and rim, ${ }^{*}=$ total iron as FeO.

(Table 6) $\mathrm{X}_{\mathrm{Na}}=\mathrm{Na} /(\mathrm{Na}+\mathrm{K}),{ }^{*}=$ total iron as $\mathrm{FeO}$.

(Table 7) $\quad \mathrm{X}_{\mathrm{Ca}}=\mathrm{Ca} /(\mathrm{Ca}+\mathrm{Na})$.

(Table 8$) *=$ total iron as $\mathrm{FeO}, \#=$ lamellae-free hematite rim.

(Table 9) $*=$ total iron as $\mathrm{FeO}$.

(Table 10) $\quad \mathrm{X}_{\mathrm{Mg}}=\mathrm{Mg} /\left(\mathrm{Mg}+\mathrm{Fe}^{2+}\right)$.

Table 1 Mineral assemblages of hematite-bearing schists in the albite-biotite zone from the Saruta-gawa area.

\begin{tabular}{|c|c|c|c|c|c|c|c|c|c|c|c|c|c|c|}
\hline \multicolumn{2}{|c|}{ Sample No. } & \multirow[b]{2}{*}{ Type } & \multirow[b]{2}{*}{ Grt } & \multirow[b]{2}{*}{ Amp } & \multirow[b]{2}{*}{ Ep } & \multirow[b]{2}{*}{$\mathrm{Chl}$} & \multirow[b]{2}{*}{ Ms } & \multirow[b]{2}{*}{ Car } & \multirow[b]{2}{*}{ Ttn } & \multirow[b]{2}{*}{ Rt } & \multirow[b]{2}{*}{$A b$} & \multirow[b]{2}{*}{ Qtz } & \multirow[b]{2}{*}{$\mathrm{Hem}$} & \multirow[b]{2}{*}{ Ap Other minerals } \\
\hline Original No. & Registration No. & & & & & & & & & & & & & \\
\hline YB108 & GSJ R76512 & BS & & + & + & + & + & & + & + & + & + & + & + Mag \\
\hline YB120 & GSJ R76513 & QS & + & + & + & + & + & & & & + & + & + & + \\
\hline ST2204 & GSJ R76514 & BS & & + & + & + & + & & & + & + & + & + & \\
\hline YB154 & GSJ R76515 & QS & + & + & + & + & + & & & & + & + & + & + Tur \\
\hline YB155 & GSJ R76516 & QS & + & + & + & + & + & & & & + & + & + & + \\
\hline YB159 & GSJ R76517 & QS & + & + & + & + & + & & & & + & + & + & + Tur \\
\hline YB35 & GSJ R76518 & BS & & + & + & + & + & & + & + & + & + & + & IIm Stp \\
\hline YB46 & GSJ R76519 & BS & & + & + & + & + & + & + & & + & + & + & + Tur \\
\hline
\end{tabular}


Table 2 Chemical compositions of amphibole.

\begin{tabular}{|c|c|c|c|c|c|c|c|c|c|c|c|c|c|c|c|c|c|c|c|c|c|c|c|}
\hline Sample No. & YB108 & YB108 & YB108 & YB108 & YB108 & YB108 & YB108 & YB108 & YB108 & YB108 & YB108 & YB108 & YB108 & YB108 & YB108 & YB108 & YB120 & YB120 & YB120 & YB120 & YB120 & YB120 & YB120 \\
\hline Rock type & BS & BS & BS & BS & BS & BS & BS & BS & BS & BS & BS & BS & BS & BS & BS & BS & QS & QS & QS & QS & QS & QS & QS \\
\hline Grain No. & $2^{\prime}$ & $2^{\prime}$ & $2^{\prime}$ & $2^{\prime}$ & $2^{\prime}$ & $2^{\prime}$ & $2^{\prime}$ & $2^{\prime}$ & 3-a & 3-a & 3-a & 3-a & 3-a & 3-a & $3-a$ & 3-a & b & b & b & b & b & c & c \\
\hline \multirow[t]{3}{*}{ Point No. } & 29 & 28 & 27 & 26 & 25 & 24 & 23 & $22 \#$ & 39 & 38 & 37 & 36 & 35 & 34 & 33 & $31 \#$ & 213 & 212 & 211 & 210 & 185\# & 209 & 208 \\
\hline & core & core & core & core & core & mantle & mantle & mantle & core & core & core & core & core & core & mantle & mantle & core & core & core & mantle & mantle & core & core \\
\hline & $\mathrm{Ba}$ & $\mathrm{Ba}$ & $\mathrm{Ba}$ & $\mathrm{Ba}$ & $\mathrm{Ba}$ & Mrb & Mrb & Mrb & $\mathrm{Ba}$ & $\mathrm{Ba}$ & $\mathrm{Ba}$ & $\mathrm{Ba}$ & $\mathrm{Ba}$ & $\mathrm{Ba}$ & $\mathrm{Ba}$ & Mrb & $\mathrm{Ba}$ & $\mathrm{Ba}$ & $\mathrm{Ba}$ & Wi & Mrb & $\mathrm{Ba}$ & $\mathrm{Ba}$ \\
\hline $\mathrm{SiO}_{2}$ & 48.5 & 48.1 & 48.4 & 48.0 & 48.4 & 55.5 & 55.6 & 55.4 & 46.8 & 45.9 & 45.9 & 46.0 & 45.7 & 45.2 & 49.2 & 54.4 & 49.5 & 49.6 & 50.0 & 53.1 & 54.9 & 50.0 & 49.3 \\
\hline $\mathrm{TiO}_{2}$ & 0.41 & 0.44 & 0.34 & 0.39 & 0.28 & 0.04 & 0.00 & 0.09 & 0.26 & 0.26 & 0.42 & 0.42 & 0.43 & 0.54 & 0.16 & 0.00 & 0.25 & 0.26 & 0.18 & 0.05 & 0.07 & 0.23 & 0.18 \\
\hline $\mathrm{Al}_{2} \mathrm{O}_{3}$ & 9.74 & 9.79 & 9.92 & 9.87 & 9.29 & 5.00 & 5.04 & 4.74 & 9.86 & 10.7 & 10.5 & 10.3 & 10.6 & 10.7 & 7.49 & 6.44 & 8.54 & 8.62 & 8.54 & 5.73 & 5.46 & 8.38 & 8.91 \\
\hline $\mathrm{Cr}_{2} \mathrm{O}_{3}$ & n.d. & n.d. & n.d. & n.d. & n.d. & n.d. & n.d. & n.d. & n.d. & n.d. & n.d. & n.d. & n.d. & n.d. & n.d. & n.d. & 0.00 & 0.02 & 0.04 & 0.03 & 0.00 & 0.00 & 0.00 \\
\hline $\mathrm{FeO}^{*}$ & 16.8 & 16.4 & 16.9 & 16.9 & 17.0 & 18.4 & 19.3 & 18.5 & 17.3 & 17.4 & 17.4 & 17.4 & 17.1 & 17.2 & 16.7 & 17.9 & 13.5 & 13.7 & 13.5 & 14.9 & 14.8 & 14.7 & 14.1 \\
\hline $\mathrm{MnO}$ & 0.27 & 0.28 & 0.21 & 0.26 & 0.25 & 0.15 & 0.20 & 0.18 & 0.27 & 0.25 & 0.21 & 0.21 & 0.29 & 0.20 & 0.22 & 0.21 & 0.67 & 0.73 & 0.60 & 0.52 & 0.47 & 0.81 & 0.71 \\
\hline $\mathrm{MgO}$ & 10.4 & 10.4 & 10.5 & 10.6 & 10.2 & 9.64 & 9.55 & 9.28 & 10.2 & 10.1 & 9.88 & 10.2 & 10.1 & 10.1 & 11.1 & 9.41 & 12.2 & 12.3 & 12.2 & 11.8 & 11.7 & 12.6 & 12.2 \\
\hline $\mathrm{CaO}$ & 6.71 & 6.76 & 6.56 & 7.07 & 6.80 & 2.14 & 2.08 & 1.87 & 7.45 & 7.45 & 7.40 & 8.01 & 8.14 & 8.30 & 7.13 & 1.97 & 6.89 & 6.69 & 6.86 & 3.80 & 3.25 & 6.47 & 6.72 \\
\hline $\mathrm{Na}_{2} \mathrm{O}$ & 4.60 & 4.61 & 4.79 & 4.52 & 4.41 & 5.88 & 5.92 & 6.11 & 3.91 & 4.08 & 4.09 & 4.03 & 3.84 & 3.84 & 3.84 & 5.91 & 4.73 & 4.99 & 4.92 & 5.70 & 6.04 & 4.76 & 5.01 \\
\hline $\mathrm{K}_{2} \mathrm{O}$ & 0.33 & 0.35 & 0.32 & 0.35 & 0.31 & 0.05 & 0.02 & 0.03 & 0.40 & 0.42 & 0.43 & 0.43 & 0.51 & 0.49 & 0.23 & 0.08 & 0.20 & 0.22 & 0.17 & 0.11 & 0.05 & 0.18 & 0.22 \\
\hline Total & 97.8 & 97.1 & 97.9 & 98.0 & 96.9 & 96.8 & 97.7 & 96.2 & 96.5 & 96.6 & 96.2 & 97.0 & 96.7 & 96.6 & 96.1 & 96.3 & 96.5 & 97.1 & 97.0 & 95.7 & 96.7 & 98.1 & 97.4 \\
\hline $\mathrm{Fe}_{2} \mathrm{O}_{3}$ & 7.65 & 7.05 & 8.22 & 7.98 & 7.35 & 9.93 & 11.4 & 9.08 & 8.66 & 9.28 & 8.40 & 7.56 & 7.30 & 7.23 & 8.24 & 10.7 & 6.01 & 6.64 & 5.28 & 8.90 & 7.78 & 10.2 & 7.30 \\
\hline $\mathrm{FeO}$ & 9.92 & 10.1 & 9.50 & 9.72 & 10.4 & 9.46 & 9.05 & 10.3 & 9.51 & 9.05 & 9.85 & 10.6 & 10.5 & 10.7 & 9.28 & 8.23 & 8.10 & 7.73 & 8.75 & 6.89 & 7.80 & 5.49 & 7.53 \\
\hline \multirow[t]{2}{*}{ New total } & 98.5 & 97.9 & 98.8 & 98.8 & 97.7 & 97.8 & 98.9 & 97.1 & 97.3 & 97.5 & 97.1 & 97.8 & 97.4 & 97.3 & 96.9 & 97.4 & 97.1 & 97.8 & 97.5 & 96.6 & 97.5 & 99.1 & 98.1 \\
\hline & \multicolumn{23}{|c|}{ Atomic ratios $(\mathrm{O}=23)$} \\
\hline $\mathrm{Si}$ & 7.000 & 6.992 & 6.967 & 6.927 & 7.054 & 7.902 & 7.849 & 7.957 & 6.873 & 6.740 & 6.777 & 6.765 & 6.742 & 6.690 & 7.199 & 7.757 & 7.166 & 7.134 & 7.205 & 7.635 & 7.791 & 7.080 & 7.080 \\
\hline${ }^{[4]} \mathrm{Al}$ & 1.000 & 1.008 & 1.033 & 1.073 & 0.946 & 0.098 & 0.151 & 0.043 & 1.127 & 1.260 & 1.223 & 1.235 & 1.258 & 1.310 & 0.801 & 0.243 & 0.834 & 0.866 & 0.795 & 0.365 & 0.209 & 0.920 & 0.920 \\
\hline${ }^{[6]} \mathrm{Al}$ & 0.657 & 0.670 & 0.650 & 0.606 & 0.650 & 0.741 & 0.688 & 0.759 & 0.580 & 0.592 & 0.604 & 0.551 & 0.585 & 0.557 & 0.490 & 0.840 & 0.623 & 0.595 & 0.655 & 0.607 & 0.704 & 0.478 & 0.589 \\
\hline $\mathrm{Ti}$ & 0.045 & 0.048 & 0.037 & 0.042 & 0.031 & 0.004 & 0.000 & 0.010 & 0.029 & 0.029 & 0.047 & 0.046 & 0.048 & 0.060 & 0.018 & 0.000 & 0.027 & 0.028 & 0.020 & 0.005 & 0.007 & 0.024 & 0.019 \\
\hline $\mathrm{Cr}$ & & & & & & & & & & & & & & & & & 0.000 & 0.002 & 0.005 & 0.003 & 0.000 & 0.000 & 0.000 \\
\hline $\mathrm{Fe}^{3+}$ & 0.831 & 0.772 & 0.890 & 0.867 & 0.807 & 1.064 & 1.210 & 0.982 & 0.957 & 1.025 & 0.933 & 0.837 & 0.810 & 0.805 & 0.908 & 1.153 & 0.654 & 0.718 & 0.573 & 0.964 & 0.830 & 1.091 & 0.789 \\
\hline $\mathrm{Fe}^{2+}$ & 1.197 & 1.222 & 1.144 & 1.173 & 1.265 & 1.127 & 1.069 & 1.240 & 1.168 & 1.112 & 1.216 & 1.304 & 1.300 & 1.324 & 1.136 & 0.982 & 0.980 & 0.930 & 1.054 & 0.828 & 0.926 & 0.650 & 0.905 \\
\hline $\mathrm{Mn}$ & 0.033 & 0.034 & 0.026 & 0.032 & 0.031 & 0.018 & 0.024 & 0.022 & 0.034 & 0.031 & 0.026 & 0.026 & 0.036 & 0.025 & 0.027 & 0.025 & 0.082 & 0.089 & 0.073 & 0.063 & 0.056 & 0.097 & 0.086 \\
\hline $\mathrm{Mg}$ & 2.238 & 2.254 & 2.253 & 2.280 & 2.216 & 2.046 & 2.010 & 1.987 & 2.233 & 2.211 & 2.175 & 2.236 & 2.221 & 2.229 & 2.421 & 2.000 & 2.633 & 2.637 & 2.621 & 2.530 & 2.475 & 2.660 & 2.612 \\
\hline $\mathrm{Ca}$ & 1.038 & 1.053 & 1.012 & 1.093 & 1.062 & 0.326 & 0.315 & 0.288 & 1.172 & 1.172 & 1.171 & 1.262 & 1.287 & 1.316 & 1.118 & 0.301 & 1.069 & 1.031 & 1.059 & 0.585 & 0.494 & 0.982 & 1.034 \\
\hline $\mathrm{Na}$ & 1.287 & 1.299 & 1.337 & 1.265 & 1.246 & 1.623 & 1.620 & 1.702 & 1.113 & 1.162 & 1.171 & 1.149 & 1.098 & 1.102 & 1.089 & 1.634 & 1.328 & 1.392 & 1.375 & 1.589 & 1.662 & 1.307 & 1.395 \\
\hline $\mathrm{K}$ & 0.061 & 0.065 & 0.059 & 0.064 & 0.058 & 0.009 & 0.004 & 0.005 & 0.075 & 0.079 & 0.081 & 0.081 & 0.096 & 0.093 & 0.043 & 0.015 & 0.037 & 0.040 & 0.031 & 0.020 & 0.009 & 0.033 & 0.040 \\
\hline Total & 15.386 & 15.417 & 15.407 & 15.422 & 15.366 & 14.959 & 14.939 & 14.995 & 15.361 & 15.413 & 15.422 & 15.492 & 15.481 & 15.511 & 15.250 & 14.950 & 15.433 & 15.463 & 15.465 & 15.195 & 15.165 & 15.321 & 15.469 \\
\hline${ }^{[B]} \mathrm{Na}$ & 0.962 & 0.947 & 0.988 & 0.907 & 0.938 & 1.623 & 1.620 & 1.702 & 0.828 & 0.828 & 0.829 & 0.738 & 0.713 & 0.684 & 0.882 & 1.634 & 0.931 & 0.969 & 0.941 & 1.415 & 1.506 & 1.018 & 0.966 \\
\hline${ }^{[\mathrm{A}]} \mathrm{Na}$ & 0.325 & 0.352 & 0.349 & 0.358 & 0.308 & 0.000 & 0.000 & 0.000 & 0.286 & 0.334 & 0.341 & 0.411 & 0.385 & 0.418 & 0.207 & 0.000 & 0.396 & 0.423 & 0.434 & 0.175 & 0.156 & 0.288 & 0.429 \\
\hline XMg & 0.651 & 0.648 & 0.663 & 0.660 & 0.637 & 0.645 & 0.653 & 0.616 & 0.657 & 0.665 & 0.641 & 0.632 & 0.631 & 0.627 & 0.681 & 0.671 & 0.729 & 0.739 & 0.713 & 0.753 & 0.728 & 0.804 & 0.743 \\
\hline $\mathrm{XFe}^{3+}$ & 0.558 & 0.535 & 0.578 & 0.589 & 0.554 & 0.590 & 0.638 & 0.564 & 0.623 & 0.634 & 0.607 & 0.603 & 0.581 & 0.591 & 0.649 & 0.579 & 0.512 & 0.547 & 0.466 & 0.614 & 0.541 & 0.695 & 0.573 \\
\hline
\end{tabular}


Table 2 Chemical compositions of amphibole (continued)

\begin{tabular}{|c|c|c|c|c|c|c|c|c|c|c|c|c|c|c|c|c|c|c|c|c|c|c|c|}
\hline Sample No. & YB120 & YB120 & YB120 & YB120 & YB120 & YB120 & YB120 & YB120 & YB120 & YB120 & YB120 & YB120 & YB120 & YB120 & YB120 & YB120 & YB120 & YB120 & YB120 & YB120 & YB120 & YB120 & YB120 \\
\hline Rock type & QS & QS & QS & QS & QS & QS & QS & QS & QS & QS & QS & QS & QS & QS & QS & QS & QS & QS & QS & QS & QS & QS & QS \\
\hline Grain No. & c & c & c & c & d & d & d & d & d & d & d & e & e & e & e & e & e & e & e & e & e & e & e \\
\hline \multirow[t]{3}{*}{ Point No. } & 207 & 217 & 218 & 219\# & 231 & 230 & 188 & 226 & 227 & 228 & $229 \#$ & 255 & 254 & 253 & 252 & 251 & 250 & 249 & 248 & 247 & 246 & 245 & 244 \\
\hline & core & mantle & mantle & mantle & core & core & core & mantle & mantle & mantle & mantle & core & core & core & core & core & core & core & core & core & core & core & core \\
\hline & $\mathrm{Ba}$ & Mrb & Wi & Mrb & $\mathrm{Ba}$ & $\mathrm{Ba}$ & $\mathrm{Ba}$ & $W_{i}$ & Mrb & Mrb & Mrb & $\mathrm{Ba}$ & $\mathrm{Ba}$ & $\mathrm{Ba}$ & $\mathrm{Ba}$ & $\mathrm{Ba}$ & $\mathrm{Ba}$ & $\mathrm{Ba}$ & $\mathrm{Ba}$ & $\mathrm{Ba}$ & $\mathrm{Ba}$ & $\mathrm{Ba}$ & $\mathrm{Ba}$ \\
\hline $\mathrm{SiO}_{2}$ & 49.9 & 55.5 & 54.0 & 55.2 & 49.2 & 49.2 & 49.6 & 53.9 & 55.8 & 55.8 & 56.7 & 50.1 & 50.3 & 50.4 & 49.2 & 49.5 & 48.9 & 49.0 & 49.5 & 49.3 & 49.7 & 49.3 & 49.5 \\
\hline $\mathrm{TiO}_{2}$ & 0.18 & 0.03 & 0.05 & 0.04 & 0.24 & 0.21 & 0.23 & 0.08 & 0.00 & 0.07 & 0.03 & 0.15 & 0.20 & 0.33 & 0.20 & 0.20 & 0.12 & 0.21 & 0.16 & 0.17 & 0.10 & 0.15 & 0.22 \\
\hline $\mathrm{Al}_{2} \mathrm{O}_{3}$ & 8.36 & 5.19 & 5.35 & 4.92 & 9.33 & 9.23 & 9.30 & 6.54 & 5.75 & 5.39 & 5.39 & 9.58 & 9.89 & 9.84 & 10.0 & 9.84 & 10.1 & 9.55 & 9.10 & 8.99 & 8.26 & 8.54 & 8.50 \\
\hline $\mathrm{Cr}_{2} \mathrm{O}_{3}$ & 0.00 & 0.00 & 0.00 & 0.06 & 0.01 & 0.04 & 0.00 & 0.00 & 0.00 & 0.05 & 0.01 & 0.03 & 0.01 & 0.00 & 0.03 & 0.05 & 0.00 & 0.03 & 0.00 & 0.00 & 0.03 & 0.00 & 0.00 \\
\hline $\mathrm{FeO}^{\star}$ & 13.9 & 15.5 & 16.2 & 16.4 & 13.8 & 13.4 & 13.9 & 14.7 & 14.1 & 14.1 & 14.9 & 13.9 & 14.2 & 13.8 & 14.1 & 13.9 & 13.4 & 13.9 & 13.6 & 14.5 & 14.4 & 13.6 & 13.7 \\
\hline $\mathrm{MnO}$ & 0.69 & .39 & 54 & 0.36 & 0.59 & 0.74 & 0.64 & 0.47 & 0.55 & 0.53 & 0.49 & 0.78 & 0.96 & 0.71 & 0.78 & 0.56 & 0.66 & 0.69 & 0.66 & 0.59 & 0.69 & 0.66 & 0.66 \\
\hline $\mathrm{MgO}$ & 12.9 & 12.1 & 11.8 & 11.4 & 12.6 & 12.4 & 12.8 & 11.9 & 11.7 & 11.9 & 11.4 & 11.7 & 11.7 & 11.6 & 11.7 & 11.4 & 11.8 & 12.3 & 12.6 & 12.3 & 12.4 & 12.4 & 12.5 \\
\hline $\mathrm{CaO}$ & 6.78 & 2.30 & 32 & 79 & .26 & 7.21 & 7.12 & 3.49 & 2.27 & 2.60 & 2.46 & 4.98 & 4.72 & 4.82 & 5.80 & 5.92 & 6.43 & 6.85 & 6.91 & 6.71 & 7.09 & 7.02 & 7.28 \\
\hline $\mathrm{Na}_{2} \mathrm{O}$ & 5.09 & 6.34 & 6.02 & 45 & 4.89 & 4.90 & 4.32 & 6.22 & 6.43 & 6.17 & 6.20 & 5.82 & 6.11 & 6.04 & 5.42 & 5.00 & 5.19 & 4.67 & 4.77 & 4.98 & 4.90 & 4.47 & 4.54 \\
\hline $\mathrm{K}_{2} \mathrm{O}$ & 0.23 & 0.07 & 0.07 & 0.02 & 0.19 & 0.22 & 0.23 & 0.11 & 0.05 & 0.04 & 0.05 & 0.20 & 0.16 & 0.17 & 0.23 & 0.21 & 0.24 & 0.18 & 0.20 & 0.19 & 0.22 & 0.19 & 0.18 \\
\hline Total & 98.0 & 97.4 & 97.4 & 96.6 & 98.1 & 97.6 & 98.1 & 97.4 & 96.7 & 96.7 & 97.6 & 97.2 & 98.3 & 97.7 & 97.5 & 96.6 & 96.8 & 97.4 & 97.5 & 97.7 & 97.8 & 96.3 & 97.1 \\
\hline $\mathrm{Fe}_{2} \mathrm{O}_{3}$ & 7.83 & 11.4 & 10.9 & 12.0 & 01 & 99 & 41 & 49 & 3 & 4 & 7.77 & 8.56 & 9.55 & 7.84 & 43 & 7.26 & 6.40 & 53 & 7.73 & 31 & 6.87 & 7.25 & 6.64 \\
\hline $\mathrm{FeO}$ & 6.86 & 5.26 & 6.41 & 5.63 & 7.49 & 8.01 & 5.44 & 7.06 & 6.70 & 6.68 & 7.91 & 6.20 & 5.61 & 6.75 & 6.52 & 7.37 & 7.64 & 6.23 & 6.64 & 7.03 & 8.21 & 7.07 & 7.73 \\
\hline \multirow[t]{2}{*}{ New total } & 98.8 & 98.6 & 98.5 & 97.9 & 98.8 & 98.2 & 99.1 & 98.3 & 97.5 & 97.5 & 98.4 & 98.1 & 99.2 & 98.5 & 98.3 & 97.3 & 97.5 & 98.2 & 98.3 & 98.6 & 98.5 & 97.1 & 97.8 \\
\hline & \multicolumn{23}{|c|}{ Atomic ratios $(\mathrm{O}=23)$} \\
\hline $\mathrm{Si}$ & 7.103 & 7.760 & 7.635 & 7.792 & 7.012 & 7.061 & 7.010 & 7.608 & 7.861 & 7.865 & 7.930 & 7.137 & 7.089 & 7.147 & 7.024 & 7.120 & 7.044 & 7.001 & 7.067 & 7.044 & 7.128 & 7.129 & 7.124 \\
\hline${ }^{[4]} \mathrm{Al}$ & 0.897 & 0.240 & 0.365 & 0.208 & 0.988 & 0.939 & 0.990 & 0.392 & 0.139 & 0.135 & 0.070 & 0.863 & 0.911 & 0.853 & 0.976 & 0.880 & 0.956 & 0.999 & 0.933 & 0.956 & 0.872 & 0.871 & 0.876 \\
\hline${ }^{[6]} \mathrm{Al}$ & 0.505 & 0.616 & 0.527 & 0.611 & 0.580 & 0.622 & 0.559 & 0.696 & 0.816 & 0.761 & 0.818 & 0.746 & 0.731 & 0.791 & 0.707 & 0.788 & 0.758 & 0.610 & 0.598 & 0.558 & 0.524 & 0.585 & 0.565 \\
\hline $\mathrm{Ti}$ & 0.019 & 0.003 & 0.005 & 0.004 & 0.026 & 0.023 & 0.024 & 0.008 & 0.000 & 0.007 & 0.003 & 0.016 & 0.021 & 0.035 & 0.021 & 0.022 & 013 & 0.023 & 0.017 & 0.018 & 0.011 & 0.016 & 0.024 \\
\hline $\mathrm{Cr}$ & 0.000 & 0.000 & 0.000 & 0.007 & 0.001 & 0.005 & 0.000 & 0.000 & 0.000 & 0.006 & 0.001 & 0.003 & 0.001 & 0.000 & 0.003 & 0.006 & 0.000 & 0.003 & 0.000 & 0.000 & 0.003 & 0.000 & 0.000 \\
\hline $\mathrm{Fe}^{3+}$ & 0.839 & 1.197 & 1.158 & 1.271 & 0.752 & 0.647 & 1.000 & 0.901 & 0.872 & 0.874 & 0.817 & 0.918 & 1.013 & 0.836 & 0.906 & 0.786 & 0.694 & 0.917 & 0.831 & 0.893 & 0.742 & 0.789 & 0.719 \\
\hline $\mathrm{Fe}^{2+}$ & 0.816 & 0.616 & 0.758 & 0.665 & 0.893 & 0.961 & 0.643 & 0.834 & 0.789 & 0.788 & 0.925 & 0.738 & 0.661 & 0.800 & 0.778 & 0.886 & 0.921 & 0.744 & 0.793 & 0.840 & 0.985 & 0.855 & 0.930 \\
\hline $\mathrm{Mn}$ & 0.083 & 0.046 & 0.065 & 0.043 & 0.071 & 0.090 & 0.077 & 0.056 & 0.066 & 0.063 & 0.058 & 0.094 & 0.115 & 0.085 & 0.094 & 0.068 & 0.081 & 0.084 & 0.080 & 0.071 & 0.084 & 0.081 & 0.080 \\
\hline $\mathrm{Mg}$ & 2.737 & 2.522 & 2.487 & 2.399 & 2.677 & 2.653 & 2.697 & 2.504 & 2.457 & 2.501 & 2.377 & 2.485 & 2.458 & 2.452 & 2.490 & 2.444 & 2.534 & 2.620 & 2.682 & 2.620 & 2.651 & 2.673 & 2.682 \\
\hline $\mathrm{Ca}$ & 1.034 & 0.345 & 0.503 & 0.271 & 1.109 & 1.109 & 1.078 & 0.528 & 0.343 & 0.393 & 0.369 & 0.760 & 0.713 & 0.732 & 0.887 & 0.912 & 0.992 & 1.049 & 1.057 & 1.027 & 1.089 & 1.088 & 1.122 \\
\hline $\mathrm{Na}$ & 1.405 & 1.719 & 1.650 & 1.765 & 1.351 & 1.363 & 1.184 & 1.702 & 1.756 & 1.686 & 1.681 & 1.608 & 1.670 & 1.661 & 1.500 & 1.394 & 1.450 & 1.294 & 1.320 & 1.380 & 1.363 & 1.253 & 1.267 \\
\hline $\mathrm{K}$ & 0.042 & 0.012 & 0.013 & 0.004 & 0.035 & 0.040 & 0.041 & 0.020 & 0.009 & 0.007 & 0.009 & 0.036 & 0.029 & 0.031 & 0.042 & 0.039 & 0.044 & 0.033 & 0.036 & 0.035 & 0.040 & 0.035 & 0.033 \\
\hline Total & 15.481 & 15.076 & 15.166 & 15.040 & 15.495 & 15.512 & 15.303 & 15.250 & 15.108 & 15.086 & 15.059 & 15.404 & 15.411 & 15.424 & 15.429 & 15.345 & 15.486 & 15.375 & 15.414 & 15.441 & 15.492 & 15.376 & 15.422 \\
\hline${ }^{[\mathrm{B}]} \mathrm{Na}$ & 0.966 & 1.655 & 1.497 & 1.729 & 0.891 & 0.891 & 0.922 & 1.472 & 1.657 & 1.607 & 1.631 & 1.240 & 1.287 & 1.268 & 1.113 & 1.088 & 1.008 & 0.951 & 0.943 & 0.973 & 0.911 & 0.912 & 0.878 \\
\hline${ }^{[\mathrm{A}]} \mathrm{Na}$ & 0.439 & 0.063 & 0.153 & 0.036 & 0.460 & 0.472 & 0.262 & 0.230 & 0.099 & 0.079 & 0.050 & 0.368 & 0.382 & 0.393 & 0.388 & 0.307 & 0.442 & 0.342 & 0.377 & 0.407 & 0.452 & 0.341 & 0.389 \\
\hline XMg & 0.770 & 0.804 & 0.767 & 0.783 & 0.750 & 0.734 & 0.808 & 0.750 & 0.757 & 0.760 & 0.720 & 0.771 & 0.788 & 0.754 & 0.762 & 0.734 & 0.734 & 0.779 & 0.772 & 0.757 & 0.729 & 0.758 & 0.743 \\
\hline $\mathrm{XFe}^{3+}$ & 0.624 & 0.660 & 0.687 & 0.675 & 0.565 & 0.510 & 0.641 & 0.564 & 0.517 & 0.535 & 0.500 & 0.552 & 0.581 & 0.514 & 0.562 & 0.499 & 0.478 & 0.601 & 0.582 & 0.616 & 0.586 & 0.574 & 0.560 \\
\hline
\end{tabular}


Table 2 Chemical compositions of amphibole (continued).

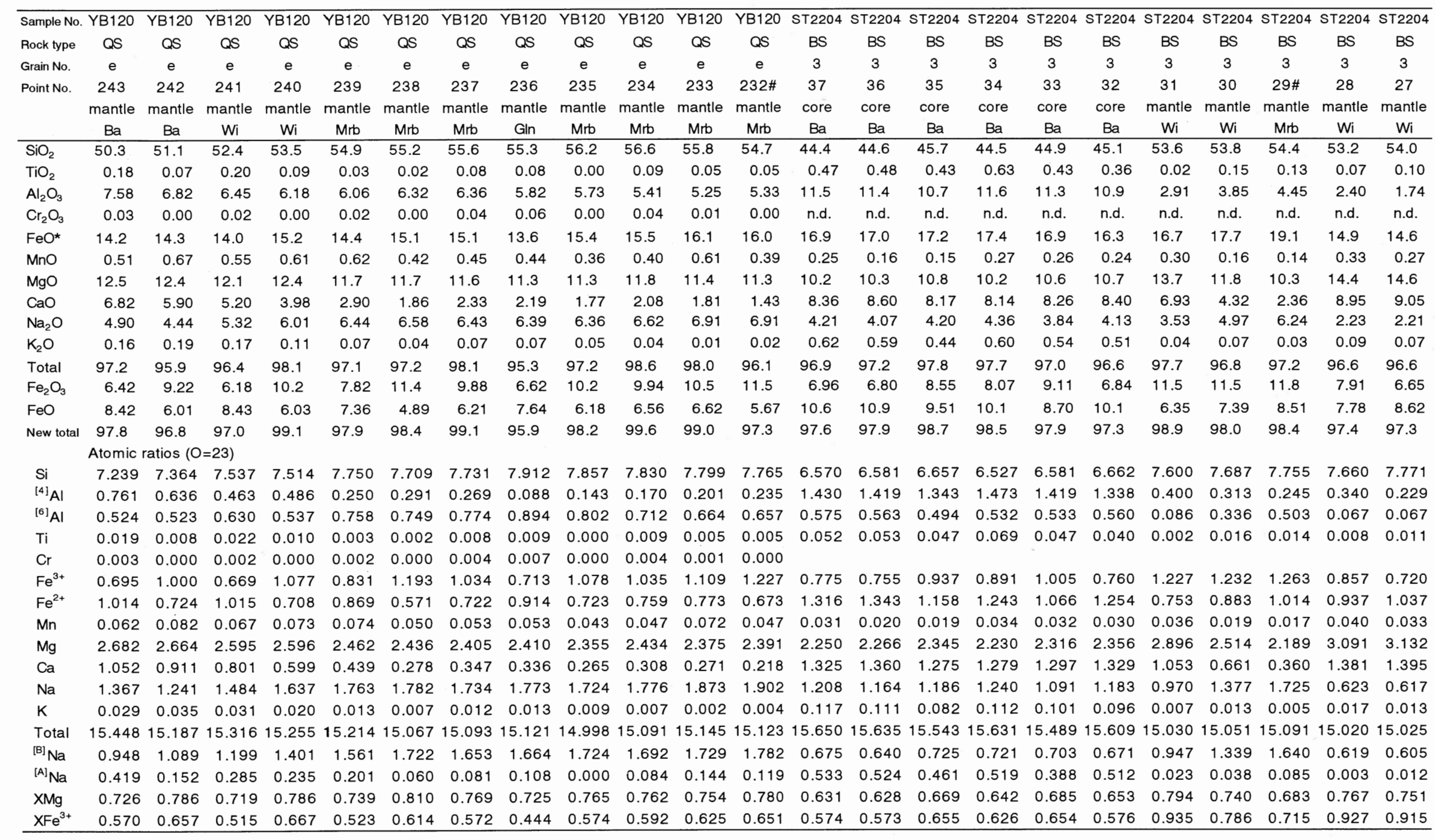


Table 2 Chemical compositions of amphibole (continued).

\begin{tabular}{|c|c|c|c|c|c|c|c|c|c|c|c|c|c|c|c|c|c|c|c|c|c|c|c|}
\hline Sample No. & YB154 & YB154 & YB154 & YB154 & YB154 & YB154 & YB154 & YB154 & YB154 & YB154 & YB154 & YB154 & YB154 & YB154 & YB154 & YB154 & YB154 & YB154 & YB154 & YB154 & YB154 & YB154 & YB154 \\
\hline Rock type & QS & QS & QS & QS & QS & QS & QS & QS & QS & QS & QS & QS & QS & QS & QS & QS & QS & QS & QS & QS & QS & QS & QS \\
\hline Grain No. & $11 \mathrm{~b}$ & $11 \mathrm{~b}$ & $11 \mathrm{~b}$ & $11 b$ & $11 \mathrm{~b}$ & $11 \mathrm{~b}$ & $11 \mathrm{~b}$ & $11 \mathrm{~b}$ & $11 \mathrm{~b}$ & $11 \mathrm{~b}$ & $11 \mathrm{~b}$ & $11 b$ & $11 \mathrm{~b}$ & $11 \mathrm{~b}$ & $11 \mathrm{~b}$ & $11 \mathrm{~b}$ & $11 b$ & $11 \mathrm{~b}$ & $11 b$ & $11 \mathrm{~b}$ & $11 \mathrm{~b}$ & 18 & 18 \\
\hline \multirow[t]{3}{*}{ Point No. } & 88 & 89 & 90 & 92 & 93 & 94 & 95 & 96 & 97 & 98 & 99 & 100 & 101 & 102 & 103 & 104 & 105 & 106 & $107 \#$ & 108 & 109 & 131 & 132 \\
\hline & core & core & core & core & core & core & core & core & core & core & core & core & mantle & mantle & mantle & mantle & mantle & mantle & mantle & mantle & mantle & core & core \\
\hline & $\mathrm{Ba}$ & $\mathrm{Ba}$ & $\mathrm{Ba}$ & $\mathrm{Ba}$ & $\mathrm{Ba}$ & $\mathrm{Ba}$ & $\mathrm{Ba}$ & $\mathrm{Ba}$ & $\mathrm{Ba}$ & $\mathrm{Ba}$ & $\mathrm{Ba}$ & $\mathrm{Ba}$ & $\mathrm{Ba}$ & $\mathrm{Ba}$ & $\mathrm{Ba}$ & $\mathrm{Ba}$ & $\mathrm{Ba}$ & Wi & Wi & Wi & Wi & $\mathrm{Ba}$ & $\mathrm{Ba}$ \\
\hline $\mathrm{SiO}_{2}$ & 44.5 & 45.3 & 44.9 & 45.2 & 45.2 & 45.0 & 45.7 & 46.9 & 45.6 & 45.6 & 45.9 & 46.1 & 46.8 & 46.7 & 48.0 & 50.0 & 50.3 & 52.6 & 53.6 & 53.1 & 52.8 & 45.4 & 45.3 \\
\hline $\mathrm{TiO}_{2}$ & 0.26 & 0.28 & 0.32 & 0.33 & 0.34 & 0.38 & 0.22 & 0.26 & 0.32 & 0.30 & 0.34 & 0.22 & 0.23 & 0.29 & 0.26 & 0.17 & 0.17 & 0.09 & 0.08 & 0.06 & 0.06 & 0.28 & 0.34 \\
\hline $\mathrm{Al}_{2} \mathrm{O}_{3}$ & 11.8 & 12.2 & 12.3 & 11.8 & 12.2 & 11.9 & 11.1 & 9.63 & 11.6 & 11.1 & 10.9 & 10.9 & 10.5 & 9.45 & 8.96 & 7.57 & 7.30 & 5.11 & 4.03 & 3.75 & 3.06 & 11.5 & 11.9 \\
\hline $\mathrm{Cr}_{2} \mathrm{O}_{3}$ & 0.02 & 0.04 & 0.02 & 0.02 & 0.03 & 0.03 & 0.00 & 0.00 & 0.00 & 0.00 & 0.00 & 0.01 & 0.00 & 0.00 & 0.02 & 0.01 & 0.01 & 0.00 & 0.02 & 0.00 & 0.00 & 0.01 & 0.04 \\
\hline $\mathrm{FeO}^{\star}$ & 17.6 & 17.4 & 17.4 & 17.3 & 15.8 & 16.8 & 17.2 & 18.5 & 17.2 & 16.6 & 17.6 & 16.9 & 17.2 & 17.1 & 17.1 & 17.5 & 17.9 & 19.5 & 20.3 & 20.7 & 19.1 & 17.8 & 16.6 \\
\hline $\mathrm{MnO}$ & 0.41 & 0.46 & 0.40 & 0.40 & 0.35 & 0.24 & 0.38 & 0.39 & 0.26 & 0.41 & 0.27 & 0.34 & 0.37 & 0.44 & 0.35 & 0.43 & 0.44 & 0.39 & 0.28 & 0.39 & 0.66 & 0.36 & 0.46 \\
\hline $\mathrm{MgO}$ & 9.46 & 9.38 & 9.80 & 9.54 & 8.96 & 9.53 & 9.48 & 9.60 & 9.80 & 9.38 & 9.72 & 9.93 & 10.3 & 10.0 & 10.1 & 10.4 & 10.2 & 9.87 & 9.88 & 10.1 & 11.5 & .47 & 9.81 \\
\hline $\mathrm{CaO}$ & 6.85 & 6.96 & 6.73 & 7.23 & 7.35 & 7.68 & 7.5 & 7.50 & 7.59 & 7.46 & 7.75 & 7.95 & 7.78 & 7.14 & 6.83 & 6.03 & 5.61 & 4.66 & 4.21 & 5.31 & 7.48 & 52 & 7.49 \\
\hline $\mathrm{Na}_{2} \mathrm{O}$ & 4.73 & 5.24 & 4.95 & 4.76 & 4.82 & 4.51 & 4.87 & 4.47 & 4.19 & 4.26 & 4.45 & 4.40 & 4.41 & 4.33 & 4.68 & 4.78 & 5.19 & 5.21 & 5.32 & 4.77 & 3.44 & 63 & 4.23 \\
\hline $\mathrm{K}_{2} \mathrm{O}$ & 0.38 & 0.37 & 0.35 & 0.33 & 0.40 & 0.44 & 0.39 & 0.33 & 0.36 & 0.33 & 0.34 & 0.36 & 0.34 & 0.21 & 0.24 & 0.20 & 0.18 & 0.13 & 0.08 & 0.10 & 0.10 & 0.34 & 0.40 \\
\hline Total & 96.0 & 97.6 & 97.2 & 96.9 & 95.5 & 96.5 & 96.9 & 97.6 & 96.9 & 95.4 & 97.3 & 97.1 & 97.9 & 95.7 & 96.5 & 97.1 & 97.3 & 97.6 & 97.8 & 98.3 & 98.2 & 97.3 & 96.6 \\
\hline $\mathrm{Fe}_{2} \mathrm{O}_{3}$ & 85 & 59 & 10.5 & 8.02 & 3.33 & 6.23 & 5.51 & 7.37 & 8.37 & 6.16 & 6.96 & 5.98 & 7.39 & 7.83 & 6.72 & 8.18 & 8.03 & 9.19 & 9.97 & 9.84 & 8.69 & 7.73 & 8.29 \\
\hline $\mathrm{FeO}$ & 8.74 & 10.6 & 7.95 & 10.1 & 12.8 & 11.2 & 12.2 & 11.9 & 9.66 & 11.1 & 11.3 & 11.5 & 10.5 & 10.1 & 11.1 & 10.1 & 10.7 & 11.2 & 11.3 & 11.8 & 11.3 & 10.8 & 9.14 \\
\hline \multirow[t]{2}{*}{ New total } & 97.0 & 98.4 & 98.2 & 97.7 & 95.8 & 97.1 & 97.4 & 98.4 & 97.8 & 96.1 & 97.9 & 97.7 & 98.6 & 96.5 & 97.3 & 97.9 & 98.1 & 98.5 & 98.8 & 99.2 & 99.1 & 98.0 & 97.4 \\
\hline & \multicolumn{23}{|c|}{ Atomic ratios $(\mathrm{O}=23)$} \\
\hline $\mathrm{Si}$ & 6.590 & 6.628 & 6.547 & 6.647 & 6.775 & 6.663 & 6.768 & 6.885 & 6.681 & 6.804 & 6.747 & 6.784 & 6.805 & 927 & 7.057 & 7.258 & 7.299 & 7.594 & 7.711 & 7.648 & 7.616 & 6.670 & 6.654 \\
\hline${ }^{[4]} \mathrm{Al}$ & 1.410 & 1.372 & 1.453 & 1.353 & 1.225 & 1.337 & 1.232 & 1.115 & 1.319 & 1.196 & 1.253 & 1.216 & 1.195 & 1.073 & 0.943 & 0.742 & 0.701 & 0.406 & 0.289 & 0.352 & 0.384 & 1.330 & 1.346 \\
\hline${ }^{[6]} \mathrm{Al}$ & 0.649 & 0.732 & 0.661 & 0.692 & 0.931 & 0.740 & 0.705 & 0.551 & 0.684 & 0.756 & 0.635 & 0.674 & 0.605 & 0.580 & 0.609 & 0.553 & 0.547 & 0.464 & 0.394 & 0.284 & 0.136 & 0.662 & 0.713 \\
\hline $\mathrm{Ti}$ & 0.029 & 0.031 & 0.035 & 0.036 & 0.038 & 0.042 & 0.024 & 0.029 & 0.035 & 0.034 & 0.038 & 0.024 & 0.025 & 0.032 & 0.029 & 0.019 & 0.019 & 0.010 & 0.009 & 0.006 & 0.007 & 0.031 & 0.038 \\
\hline $\mathrm{Cr}$ & 0.002 & 0.005 & 0.002 & 0.002 & 0.004 & 0.004 & 0.000 & 0.000 & 0.000 & 0.000 & 0.000 & 0.001 & 0.000 & 0.000 & 0.002 & 0.001 & 0.001 & 0.000 & 0.002 & 0.000 & 0.000 & 0.001 & 0.005 \\
\hline $\mathrm{Fe}^{3+}$ & 1.097 & 0.835 & 1.152 & 0.888 & 0.375 & 0.694 & 0.614 & 0.814 & 0.923 & 0.691 & 0.770 & 0.663 & 0.809 & 0.874 & 0.743 & 0.894 & 0.877 & 0.998 & 1.080 & 1.066 & 0.943 & 0.855 & 0.916 \\
\hline $\mathrm{Fe}^{2+}$ & 1.082 & 1.294 & 0.970 & 1.240 & 1.605 & 1.387 & 1.516 & 1.457 & 1.184 & 1.380 & 1.394 & 1.417 & 1.283 & 1.248 & 1.359 & 1.231 & 1.295 & 1.356 & 1.363 & 1.427 & 1.361 & 1.333 & 1.123 \\
\hline $\mathrm{Mn}$ & 0.051 & 0.057 & 0.049 & 0.050 & 0.044 & 0.030 & 0.048 & 0.048 & 0.032 & 0.052 & 0.034 & 0.042 & 0.046 & 0.055 & 0.044 & 0.053 & 0.054 & 0.048 & 0.034 & 0.048 & 0.081 & 0.045 & 0.057 \\
\hline $\mathrm{Mg}$ & 2.088 & 2.046 & 2.130 & 2.091 & 2.002 & 2.104 & 2.093 & 2.101 & 2.141 & 2.087 & 2.130 & 2.178 & 2.233 & 2.211 & 2.214 & 2.250 & 2.207 & 2.124 & 2.119 & 2.169 & 2.473 & 2.074 & 2.148 \\
\hline $\mathrm{Ca}$ & 1.087 & 1.091 & 1.051 & 1.139 & 1.180 & 1.218 & 1.196 & 1.180 & 1.192 & 1.193 & 1.221 & 1.253 & 1.212 & 1.135 & 1.076 & 0.938 & 0.872 & 0.721 & 0.649 & 0.819 & 1.156 & 1.184 & 1.179 \\
\hline $\mathrm{Na}$ & 1.358 & 1.487 & 1.399 & 1.357 & 1.401 & 1.295 & 1.398 & 1.272 & 1.190 & 1.232 & 1.268 & 1.255 & 1.243 & 1.245 & 1.334 & 1.345 & 1.460 & 1.458 & 1.484 & 1.332 & 0.962 & 1.319 & 1.205 \\
\hline$K$ & 0.072 & 0.069 & 0.065 & 0.062 & 0.076 & 0.083 & 0.074 & 0.062 & 0.067 & 0.063 & 0.064 & 0.068 & 0.063 & 0.040 & 0.045 & 0.037 & 0.033 & 0.024 & 0.015 & 0.018 & 0.018 & 0.064 & 0.075 \\
\hline Total & 15.517 & 15.647 & 15.516 & 15.558 & 15.658 & 15.596 & 15.668 & 15.514 & 15.449 & 15.488 & 15.553 & 15.576 & 15.519 & 15.420 & 15.455 & 15.320 & 15.366 & 15.203 & 15.147 & 15.170 & 15.136 & 15.566 & 15.458 \\
\hline${ }^{[\mathrm{B}]} \mathrm{Na}$ & 0.913 & 0.909 & 0.949 & 0.861 & 0.820 & 0.782 & 0.804 & 0.820 & 0.808 & 0.807 & 0.779 & 0.747 & 0.788 & 0.865 & 0.924 & 1.062 & 1.128 & 1.279 & 1.351 & 1.181 & 0.844 & 0.816 & 0.821 \\
\hline${ }^{[\mathrm{A}]} \mathrm{Na}$ & 0.445 & 0.578 & 0.451 & 0.496 & 0.581 & 0.513 & 0.595 & 0.452 & 0.382 & 0.425 & 0.489 & 0.509 & 0.455 & 0.380 & 0.410 & 0.283 & 0.332 & 0.179 & 0.133 & 0.151 & 0.118 & 0.503 & 0.383 \\
\hline $\mathrm{XMg}$ & 0.659 & 0.613 & 0.687 & 0.628 & 0.555 & 0.603 & 0.580 & 0.590 & 0.644 & 0.602 & 0.604 & 0.606 & 0.635 & 0.639 & 0.620 & 0.646 & 0.630 & 0.610 & 0.609 & 0.603 & 0.645 & 0.609 & 0.657 \\
\hline $\mathrm{XFe}^{3+}$ & 0.628 & 0.533 & 0.635 & 0.562 & 0.287 & 0.484 & 0.466 & 0.596 & 0.574 & 0.478 & 0.548 & 0.496 & 0.572 & 0.601 & 0.550 & 0.618 & 0.616 & 0.683 & 0.733 & 0.790 & 0.874 & 0.564 & 0.562 \\
\hline
\end{tabular}


Table 2 Chemical compositions of amphibole (continued).

\begin{tabular}{|c|c|c|c|c|c|c|c|c|c|c|c|c|c|c|c|c|c|c|c|c|c|c|c|}
\hline Sample No. & YB154 & YB154 & YB154 & YB154 & YB154 & $\begin{array}{l}\text { YB154 } \\
\end{array}$ & YB154 & YB154 & YB154 & YB154 & YB154 & YB154 & YB154 & YB155 & YB155 & YB155 & YB155 & YB155 & YB155 & YB155 & YB155 & YB155 & YB155 \\
\hline Rock type & QS & QS & QS & QS & QS & QS & QS & QS & QS & QS & QS & QS & QS & QS & QS & QS & QS & QS & QS & QS & QS & QS & QS \\
\hline Grain No. & 18 & 18 & 18 & 18 & 18 & 18 & 18 & 18 & 18 & 18 & 18 & 18 & 18 & 27 & 27 & 27 & 27 & 27 & 27 & 27 & 27 & 27 & 27 \\
\hline \multirow[t]{3}{*}{ Point No. } & 133 & 134 & 135 & 136 & 137 & 138 & 139 & 140 & 141 & 142 & 143 & 144 & $145 \#$ & 39 & 41 & 42 & 43 & 44 & 45 & 46 & 47 & 49 & 50 \\
\hline & core & core & core & core & core & core & core & core & core & mantle & mantle & mantle & mantle & core & core & core & core & core & core & core & core & core & core \\
\hline & $\mathrm{Ba}$ & $\mathrm{Ba}$ & $\mathrm{Ba}$ & $\mathrm{Ba}$ & $\mathrm{Ba}$ & $\mathrm{Ba}$ & $\mathrm{Ba}$ & $\mathrm{Ba}$ & $\mathrm{Ba}$ & $\mathrm{Ba}$ & $\mathrm{Ba}$ & Wi & Wi & $\mathrm{Ba}$ & $\mathrm{Ba}$ & $\mathrm{Ba}$ & $\mathrm{Ba}$ & $\mathrm{Ba}$ & $\mathrm{Ba}$ & $\mathrm{Ba}$ & $\mathrm{Ba}$ & $\mathrm{Ba}$ & $\mathrm{Ba}$ \\
\hline $\mathrm{SiO}_{2}$ & 44.6 & 45.2 & 45.8 & 45.6 & 45.8 & 46.6 & 46.7 & 46.9 & 46.8 & 48.1 & 47.4 & 52.8 & 53.1 & 47.1 & 47.3 & 47.8 & 47.9 & 47.8 & 48.6 & 46.6 & 46.5 & 47.1 & 47.1 \\
\hline $\mathrm{TiO}_{2}$ & 0.31 & 0.39 & 0.29 & 0.30 & 0.26 & 0.31 & 0.25 & 0.31 & 0.23 & 0.24 & 0.24 & 0.00 & 0.02 & 0.26 & 0.36 & 0.17 & 0.20 & 0.22 & 0.29 & 0.28 & 0.25 & 0.21 & 0.18 \\
\hline $\mathrm{Al}_{2} \mathrm{O}_{3}$ & 11.4 & 11.1 & 11.5 & 11.5 & 11.1 & 10.8 & 10.5 & 10.2 & 9.99 & 9.06 & 9.71 & 4.82 & 4.70 & 10.7 & 10.4 & 10.6 & 10.5 & 10.5 & 8.82 & 10.5 & 10.9 & 10.4 & 10.9 \\
\hline $\mathrm{Cr}_{2} \mathrm{O}_{3}$ & 0.00 & 0.00 & 0.01 & 0.00 & 0.00 & 0.04 & 0.01 & 0.01 & 0.00 & 0.04 & 0.01 & 0.01 & 0.01 & 0.00 & 0.07 & 0.00 & 0.00 & 0.02 & 0.00 & 0.00 & 0.00 & 0.05 & 0.03 \\
\hline $\mathrm{FeO}^{\star}$ & 17.0 & 17.3 & 17.4 & 17.4 & 16.2 & 17.0 & 16.7 & 16.1 & 16.9 & 17.3 & 17.1 & 17.1 & 17.4 & 11.8 & 11.2 & 11.6 & 11.5 & 11.5 & 13.2 & 11.3 & 11.1 & 12.1 & 11.8 \\
\hline $\mathrm{MnO}$ & 0.41 & 0.49 & 0.35 & 0.34 & 0.33 & 0.41 & 0.30 & 0.43 & 0.40 & 0.44 & 0.37 & 0.38 & 0.22 & 1.00 & 1.07 & 1.11 & 1.02 & 1.02 & 1.01 & 1.14 & 1.00 & 1.16 & 1.13 \\
\hline $\mathrm{MgO}$ & 9.84 & 9.98 & 9.86 & 9.87 & 9.38 & 10.3 & 10.1 & 9.61 & 9.98 & 10.7 & 8.97 & 11.3 & 10.9 & 13.6 & 13.2 & 13.3 & 13.6 & 13.5 & 12.9 & 13.4 & 13.3 & 13.9 & 13.4 \\
\hline $\mathrm{CaO}$ & 7.91 & 7.62 & 7.67 & 7.62 & 7.84 & 7.78 & 7.73 & 7.42 & 7.54 & 6.67 & 7.25 & 5.66 & 5.21 & 6.93 & 6.93 & 7.02 & 7.19 & 6.75 & 6.41 & 6.97 & 7.15 & 6.84 & 7.13 \\
\hline $\mathrm{Na}_{2} \mathrm{O}$ & 4.50 & 4.60 & 4.63 & 4.73 & 4.56 & 4.55 & 4.43 & 4.39 & 4.61 & 4.69 & 4.59 & 4.57 & 4.71 & 4.56 & 4.54 & 4.58 & 4.72 & 4.71 & .84 & 4.73 & 4.82 & 4.38 & 4.73 \\
\hline $\mathrm{K}_{2} \mathrm{O}$ & 0.40 & 0.35 & 0.36 & 0.35 & 0.36 & 0.28 & 0.25 & 0.28 & 0.27 & 0.24 & 0.27 & 0.12 & 0.08 & 0.35 & 0.36 & 0.39 & 0.37 & 0.33 & 0.34 & 0.36 & 0.31 & 0.34 & 0.38 \\
\hline Total & 96.4 & 97.0 & 97.9 & 97.7 & 95.8 & 98.1 & 97.0 & 95.7 & 96.7 & 97.5 & 95.9 & 96.8 & 96.4 & 96.3 & 95.4 & 96.6 & 97.0 & 96.4 & 96.4 & 95.3 & 95.3 & 96.5 & 96.8 \\
\hline $\mathrm{Fe}_{2} \mathrm{O}_{3}$ & 7.09 & 8.16 & 7.51 & 7.53 & 3.32 & 7.22 & 6.11 & 4.35 & 5.81 & 9.52 & 3.30 & 7.93 & 7.64 & 11.3 & 8.83 & 9.38 & 9.00 & 10.1 & 9.83 & 9.63 & 8.50 & 13.5 & 10.1 \\
\hline $\mathrm{FeO}$ & 10.6 & 9.96 & 10.6 & 10.6 & 13.2 & 10.5 & 11.2 & 12.2 & 11.7 & 8.73 & 14.1 & 9.97 & 10.5 & 1.64 & 3.26 & 3.16 & 3.40 & 2.41 & 4.35 & 2.63 & 3.45 & 0.00 & 2.73 \\
\hline \multirow[t]{2}{*}{ New total } & 97.1 & 97.9 & 98.6 & 98.4 & 96.2 & 98.8 & 97.6 & 96.1 & 97.3 & 98.4 & 96.2 & 97.6 & 97.1 & 97.4 & 96.3 & 97.5 & 97.9 & 97.4 & 7.4 & 96.2 & 96.2 & 97.9 & 97.8 \\
\hline & \multicolumn{23}{|c|}{ Atomic ratios $(\mathrm{O}=23)$} \\
\hline $\mathrm{Si}$ & 6.626 & 6.652 & 6.680 & 6.666 & 6.852 & 6.768 & 6.856 & 6.983 & 6.906 & 6.965 & 7.081 & 7.630 & 7.700 & 6.736 & 6.845 & 6.837 & 6.829 & 6.831 & 6.994 & 6.763 & 6.755 & 6.702 & 6.735 \\
\hline${ }^{[4]} \mathrm{Al}$ & 1.374 & 1.348 & 1.320 & 1.334 & 1.148 & 1.232 & 1.144 & 1.017 & 1.094 & 1.035 & 0.919 & 0.370 & 0.300 & 1.264 & 1.155 & 1.163 & 1.171 & 1.169 & 1.006 & 1.237 & 1.245 & 1.298 & 1.265 \\
\hline${ }^{[6]} \mathrm{Al}$ & 0.622 & 0.577 & 0.657 & 0.647 & 0.810 & 0.616 & 0.673 & 0.772 & 0.643 & 0.511 & 0.791 & 0.451 & 0.503 & 0.540 & 0.619 & 0.624 & 0.594 & 0.600 & 0.490 & 0.559 & 0.621 & 0.446 & 0.572 \\
\hline $\mathrm{Ti}$ & 0.035 & 0.043 & 0.032 & 0.033 & 0.029 & 0.034 & 0.028 & 0.035 & 0.026 & 0.026 & 0.027 & 0.000 & 0.002 & 0.028 & 0.039 & 0.018 & 0.021 & 0.024 & 0.031 & 0.031 & 0.027 & 0.022 & 0.019 \\
\hline $\mathrm{Cr}$ & 0.000 & 0.000 & 0.001 & 0.000 & 0.000 & 0.005 & 0.001 & 0.001 & 0.000 & 0.005 & 0.001 & 0.001 & 0.001 & 0.000 & 0.008 & 0.000 & 0.000 & 0.002 & 0.000 & 0.000 & 0.000 & 0.006 & 0.003 \\
\hline $\mathrm{Fe}^{3+}$ & 0.792 & 0.904 & 0.824 & 0.829 & 0.374 & 0.789 & 0.675 & 0.487 & 0.646 & 1.037 & 0.371 & 0.862 & 0.833 & 1.216 & 0.961 & 1.010 & 0.966 & 1.087 & 1.065 & 1.052 & 0.929 & 1.440 & 1.084 \\
\hline $\mathrm{Fe}^{2+}$ & 1.320 & 1.226 & 1.299 & 1.298 & 1.653 & 1.275 & 1.376 & 1.518 & 1.440 & 1.058 & 1.766 & 1.205 & 1.277 & 0.196 & 0.394 & 0.378 & 0.406 & 0.288 & 0.524 & 0.319 & 0.420 & 0.000 & 0.327 \\
\hline $\mathrm{Mn}$ & 0.052 & 0.061 & 0.043 & 0.042 & 0.042 & 0.050 & 0.037 & 0.054 & 0.050 & 0.054 & 0.047 & 0.047 & 0.027 & 0.121 & 0.131 & 0.134 & 0.123 & 0.123 & 0.123 & 0.140 & 0.123 & 0.140 & 0.137 \\
\hline $\mathrm{Mg}$ & 2.179 & 2.190 & 2.144 & 2.151 & 2.092 & 2.230 & 2.211 & 2.133 & 2.195 & 2.310 & 1.998 & 2.434 & 2.356 & 2.900 & 2.848 & 2.836 & 2.891 & 2.876 & 2.767 & 2.899 & 2.880 & 2.949 & 2.857 \\
\hline $\mathrm{Ca}$ & 1.259 & 1.201 & 1.199 & 1.193 & 1.257 & 1.211 & 1.216 & 1.184 & 1.192 & 1.035 & 1.160 & 0.876 & 0.809 & 1.062 & 1.074 & 1.076 & 1.098 & 1.034 & 0.988 & 1.084 & 1.113 & 1.043 & 1.092 \\
\hline $\mathrm{Na}$ & 1.296 & 1.313 & 1.309 & 1.341 & 1.323 & 1.281 & 1.261 & 1.267 & 1.319 & 1.317 & 1.330 & 1.281 & 1.324 & 1.265 & 1.274 & 1.270 & 1.305 & 1.305 & 1.350 & 1.331 & 1.358 & 1.208 & 1.311 \\
\hline $\mathrm{K}$ & 0.076 & 0.066 & 0.067 & 0.065 & 0.069 & 0.052 & 0.047 & 0.053 & 0.051 & 0.044 & 0.051 & 0.022 & 0.015 & 0.064 & 0.066 & 0.071 & 0.067 & 0.060 & 0.062 & 0.067 & 0.057 & 0.062 & 0.069 \\
\hline Total & 15.631 & 15.580 & 15.575 & 15.599 & 15.648 & 15.544 & 15.524 & 15.504 & 15.562 & 15.396 & 15.541 & 15.179 & 15.149 & 15.390 & 15.415 & 15.417 & 15.470 & 15.399 & 15.401 & 15.481 & 15.528 & 15.316 & 15.473 \\
\hline${ }^{[B]} \mathrm{Na}$ & 0.741 & 0.799 & 0.801 & 0.807 & 0.743 & 0.789 & 0.784 & 0.816 & 0.808 & 0.965 & 0.840 & 1.124 & 1.191 & 0.938 & 0.926 & 0.924 & 0.902 & 0.966 & 1.012 & 0.916 & 0.887 & 0.954 & 0.908 \\
\hline${ }^{[\mathrm{A}]} \mathrm{Na}$ & 0.555 & 0.514 & 0.508 & 0.534 & 0.580 & 0.492 & 0.477 & 0.451 & 0.511 & 0.351 & 0.490 & 0.157 & 0.134 & 0.326 & 0.348 & 0.346 & 0.403 & 0.339 & 0.339 & 0.415 & 0.470 & 0.254 & 0.404 \\
\hline $\mathrm{XMg}$ & 0.623 & 0.641 & 0.623 & 0.624 & 0.559 & 0.636 & 0.616 & 0.584 & 0.604 & 0.686 & 0.531 & 0.669 & 0.649 & 0.937 & 0.878 & 0.882 & 0.877 & 0.909 & 0.841 & 0.901 & 0.873 & 1.000 & 0.897 \\
\hline $\mathrm{XFe}^{3+}$ & 0.560 & 0.610 & 0.556 & 0.562 & 0.316 & 0.562 & 0.501 & 0.387 & 0.501 & 0.670 & 0.319 & 0.656 & 0.624 & 0.693 & 0.608 & 0.618 & 0.619 & 0.644 & 0.685 & 0.653 & 0.599 & 0.763 & 0.654 \\
\hline
\end{tabular}


Table 2 Chemical compositions of amphibole (continued).

\begin{tabular}{|c|c|c|c|c|c|c|c|c|c|c|c|c|c|c|c|c|c|c|c|c|c|c|c|}
\hline Sample No. & YB155 & YB155 & YB155 & YB155 & YB155 & YB155 & YB155 & YB155 & YB155 & YB155 & YB155 & YB159 & YB159 & YB159 & YB159 & YB159 & YB159 & YB159 & YB159 & YB159 & YB159 & YB159 & $\overline{\text { YB159 }}$ \\
\hline Rock type & QS & QS & QS & QS & QS & QS & QS & QS & $Q S$ & QS & QS & QS & QS & QS & QS & QS & QS & QS & QS & QS & QS & QS & QS \\
\hline Grain No. & 27 & 27 & 27 & 27 & 27 & 27 & 27 & 27 & 27 & 27 & 27 & 17 & 17 & 17 & 17 & 17 & 17 & 17 & 17 & 17 & 17 & 17 & 17 \\
\hline \multirow[t]{3}{*}{ Point No. } & 51 & 52 & 53 & 54 & 55 & 56 & 57 & 58 & 59 & 60 & 61\# & 29 & 30 & 32 & 33 & 34 & 35 & 37 & 38 & 39 & 40 & 41 & 43 \\
\hline & core & core & core & core & mantle & mantle & mantle & mantle & mantle & mantle & mantle & core & core & core & core & core & core & core & core & mantle & mantle & mantle & mantle \\
\hline & $\mathrm{Ba}$ & $\mathrm{Ba}$ & $\mathrm{Ba}$ & $\mathrm{Ba}$ & $\mathrm{Ba}$ & $\mathrm{Ba}$ & $\mathrm{Ba}$ & $W_{i}$ & Wi & Wi & Wi & $\mathrm{Ba}$ & $\mathrm{Ba}$ & $\mathrm{Ba}$ & $\mathrm{Ba}$ & $\mathrm{Ba}$ & $\mathrm{Ba}$ & $\mathrm{Ba}$ & $\mathrm{Ba}$ & $\mathrm{Ba}$ & $\mathrm{Ba}$ & $\mathrm{Ba}$ & $\mathrm{Ba}$ \\
\hline $\mathrm{SiO}_{2}$ & 46.9 & 47.4 & 47.5 & 47.8 & 50.1 & 50.5 & 51.7 & 53.1 & 53.0 & 53.2 & 53.2 & 49.0 & 48.9 & 48.9 & 48.1 & 49.0 & 49.5 & 49.2 & 49.1 & 49.6 & 50.1 & 50.5 & $\begin{array}{l}51.3 \\
\end{array}$ \\
\hline $\mathrm{TiO}_{2}$ & 0.23 & 0.27 & 0.21 & 0.17 & 0.13 & 0.19 & 0.11 & 0.08 & 0.04 & 0.00 & 0.04 & 0.23 & 0.20 & 0.22 & 0.17 & 0.22 & 0.17 & 0.23 & 0.13 & 0.18 & 0.16 & 0.12 & 0.12 \\
\hline $\mathrm{Al}_{2} \mathrm{O}_{3}$ & 9.73 & 10.6 & 10.5 & 10.3 & 8.29 & 7.69 & 6.39 & 4.14 & 4.20 & 3.70 & 3.59 & 9.12 & 9.71 & 8.96 & 8.79 & 8.78 & 8.09 & 7.33 & 7.52 & 7.13 & 6.77 & 6.52 & 5.86 \\
\hline $\mathrm{Cr}_{2} \mathrm{O}_{3}$ & 0.00 & 0.00 & 0.00 & 0.01 & 0.06 & 0.04 & 0.01 & 0.00 & 0.03 & 0.00 & 0.02 & 0.02 & 0.00 & 0.03 & 0.01 & 0.00 & 0.00 & 0.00 & 0.00 & 0.04 & 0.01 & 0.02 & 0.00 \\
\hline $\mathrm{FeO}^{*}$ & 12.3 & 11.6 & 11.6 & 12.4 & 11.4 & 11.6 & 12.4 & 13.4 & 14.2 & 14.8 & 15.5 & 16.7 & 17.0 & 17.2 & 17.4 & 16.5 & 16.8 & 15.9 & 16.7 & 16.4 & 16.5 & 16.5 & 16.5 \\
\hline $\mathrm{MnO}$ & 0.95 & 0.99 & 1.07 & 0.75 & .70 & 0.69 & 0.64 & 0.66 & 0.63 & 0.82 & 0.72 & 0.46 & 0.49 & 0.57 & 0.40 & 0.50 & 0.56 & 0.43 & 0.49 & 0.47 & 0.46 & 0.45 & 0.48 \\
\hline $\mathrm{MgO}$ & 13.1 & 13.3 & 13.1 & 12.9 & 14.4 & 14.2 & 14.2 & 13.5 & 13.7 & 13.3 & 12.9 & 9.91 & 9.40 & 9.95 & 9.98 & 10.8 & 10.5 & 11.5 & 11.1 & 11.5 & 11.4 & 11.3 & 11.7 \\
\hline $\mathrm{CaO}$ & 6.87 & 7.02 & .06 & .54 & 65 & 6.80 & .15 & 7.01 & 7.01 & 6.45 & 6.50 & 5.62 & 4.87 & 5.12 & 6.38 & 6.72 & 6.03 & 7.21 & 7.20 & 7.14 & 7.03 & 6.81 & 6.26 \\
\hline $\mathrm{Na}_{2} \mathrm{O}$ & 4.73 & 4.73 & 4.86 & 4.71 & 4.22 & 4.33 & 3.81 & 3.79 & 3.96 & 3.29 & 3.66 & 5.13 & 5.74 & 5.54 & 4.52 & 4.81 & 4.67 & 4.33 & 4.43 & 3.60 & 3.62 & 4.40 & 4.41 \\
\hline $\mathrm{K}_{2} \mathrm{O}$ & 0.37 & 0.36 & 0.33 & 0.25 & 0.16 & 0.12 & 0.14 & 0.12 & 0.08 & 0.13 & 0.09 & 0.24 & 0.27 & 0.20 & 0.30 & 0.29 & 0.20 & 0.23 & 0.23 & 0.18 & 0.19 & 0.16 & 0.11 \\
\hline Total & 95.2 & 96.3 & 96.2 & 96.8 & 97.1 & 96.2 & 96.6 & 95.8 & 96.9 & 95.7 & 96.2 & 96.4 & 96.6 & 96.7 & 96.1 & 97.6 & 96.5 & 96.4 & 96.9 & 96.2 & 96.2 & 96.8 & 96.7 \\
\hline $\mathrm{Fe}_{2} \mathrm{O}_{3}$ & 9.56 & 9.04 & 8.05 & 7.08 & 90 & 9.03 & 8.81 & 6.16 & 7.86 & 10.7 & 36 & 7.47 & 7.55 & 9.01 & 8.38 & 7.22 & 8.54 & 6.80 & 7.09 & 9.52 & 9.00 & 6.91 & 8.75 \\
\hline $\mathrm{FeO}$ & 3.70 & 3.47 & 4.35 & 6.03 & 4.29 & 3.47 & 4.47 & 7.86 & 7.13 & 5.18 & 7.07 & 9.98 & 10.2 & 9.09 & 9.86 & 10.0 & 9.12 & 9.78 & 10.3 & 7.83 & 8.40 & 10.3 & 8.63 \\
\hline \multirow[t]{2}{*}{ New total } & 96.1 & 97.2 & 97.0 & 97.5 & 97.9 & 97.1 & 97.4 & 96.4 & 97.6 & 96.8 & 97.2 & 97.2 & 97.3 & 97.6 & 96.9 & 98.3 & 97.4 & 97.0 & 97.6 & 97.2 & 97.1 & 97.5 & 97.6 \\
\hline & \multicolumn{23}{|c|}{ Atomic ratios $(\mathrm{O}=23)$} \\
\hline $\mathrm{Si}$ & 6.838 & 6.812 & 6.848 & 6.881 & 7.110 & 7.199 & 7.353 & 7.676 & 7.585 & 7.645 & 661 & 7.152 & 7.131 & 7.115 & 7.076 & 7.091 & 7.203 & 7.201 & 7.173 & 7.210 & 7.286 & 7.350 & 7.415 \\
\hline${ }^{[4]} \mathrm{Al}$ & 1.162 & 1.188 & 1.152 & 1.119 & 0.890 & 0.801 & 0.647 & 0.324 & 0.415 & 0.355 & 0.339 & 0.848 & 0.869 & 0.885 & 0.924 & 0.909 & 0.797 & 0.799 & 0.827 & 0.790 & 0.714 & 0.650 & 0.585 \\
\hline${ }^{[6]} \mathrm{Al}$ & 0.510 & 0.607 & 0.632 & 0.628 & 0.496 & 0.491 & 0.424 & 0.381 & 0.293 & 0.272 & 0.270 & 0.721 & 0.800 & 0.651 & 0.601 & 0.588 & 0.590 & 0.466 & 0.467 & 0.432 & 0.446 & 0.469 & 0.413 \\
\hline $\mathrm{Ti}$ & 0.025 & 0.029 & 0.023 & 0.018 & 0.014 & 0.020 & 0.012 & 0.009 & 0.004 & 0.000 & 0.004 & 0.025 & 0.022 & 0.024 & 0.019 & 0.024 & 0.019 & 0.025 & 0.014 & 0.020 & 0.017 & 0.013 & 0.013 \\
\hline $\mathrm{Cr}$ & 0.000 & 0.000 & 0.000 & 0.001 & 0.007 & 0.005 & 0.001 & 0.000 & 0.003 & 0.000 & 0.002 & 0.002 & 0.000 & 0.003 & 0.001 & 0.000 & 0.000 & 0.000 & 0.000 & 0.005 & 0.001 & 0.002 & 0.000 \\
\hline $\mathrm{Fe}^{3+}$ & 1.049 & 0.977 & 0.874 & 0.767 & 0.844 & 0.969 & 0.943 & 0.670 & 0.846 & 1.156 & 1.015 & 0.820 & 0.829 & 0.986 & 0.927 & 0.786 & 0.935 & 0.749 & 0.780 & 1.042 & 0.985 & 0.757 & 0.952 \\
\hline $\mathrm{Fe}^{2+}$ & 0.451 & 0.417 & 0.525 & 0.726 & 0.509 & 0.414 & 0.532 & 0.950 & 0.853 & 0.623 & .852 & 1.218 & 1.244 & 1.107 & 1.213 & 1.210 & 1.110 & 1.197 & 1.261 & 0.952 & 1.022 & 1.251 & 1.043 \\
\hline $\mathrm{Mn}$ & 0.117 & 0.121 & 0.131 & 0.091 & 0.084 & 0.083 & 0.077 & 0.081 & 0.076 & 0.100 & 0.088 & 0.057 & 0.061 & 0.070 & 0.050 & 0.061 & 0.069 & 0.053 & 0.061 & 0.058 & 0.057 & 0.055 & 0.059 \\
\hline $\mathrm{Mg}$ & 2.847 & 2.849 & 2.816 & 2.768 & 3.046 & 3.018 & 3.011 & 2.909 & 2.923 & 2.849 & 2.769 & 2.156 & 2.044 & 2.158 & 2.189 & 2.330 & 2.278 & 2.509 & 2.417 & 2.492 & 2.472 & 2.452 & 2.521 \\
\hline $\mathrm{Ca}$ & 1.073 & 1.081 & 1.091 & 1.163 & 1.163 & $1.039^{\circ}$ & 1.090 & 1.086 & 1.075 & 0.993 & 1.003 & 0.879 & 0.761 & 0.798 & 1.006 & 1.042 & 0.940 & 1.131 & 1.127 & 1.112 & 1.095 & 1.062 & 0.969 \\
\hline $\mathrm{Na}$ & 1.337 & 1.318 & 1.359 & 1.315 & 1.161 & 1.197 & 1.051 & 1.062 & 1.099 & 0.917 & 1.022 & 1.452 & 1.623 & 1.563 & 1.289 & 1.350 & 1.318 & 1.229 & 1.255 & 1.015 & 1.021 & 1.242 & 1.236 \\
\hline $\mathrm{K}$ & 0.069 & 0.066 & 0.061 & 0.046 & 0.029 & 0.022 & 0.025 & 0.022 & 0.015 & 0.024 & 0.017 & 0.045 & 0.050 & 0.037 & 0.056 & 0.054 & 0.037 & 0.043 & 0.043 & 0.033 & 0.035 & 0.030 & 0.020 \\
\hline Total & 15.479 & 15.465 & 15.510 & 15.523 & 15.353 & 15.257 & 15.166 & 15.170 & 15.188 & 14.934 & 15.041 & 15.375 & 15.434 & 15.398 & 15.351 & 15.445 & 15.295 & 15.402 & 15.425 & 15.160 & 15.151 & 15.333 & 15.226 \\
\hline${ }^{[\mathrm{B}]} \mathrm{Na}$ & 0.927 & 0.919 & 0.909 & 0.837 & 0.837 & 0.961 & 0.910 & 0.914 & 0.925 & 0.917 & 0.997 & 1.121 & 1.239 & 1.202 & 0.994 & 0.958 & 1.060 & 0.869 & 0.873 & 0.888 & 0.905 & 0.938 & 1.031 \\
\hline${ }^{[\mathrm{A}]} \mathrm{Na}$ & 0.410 & 0.399 & 0.449 & 0.477 & 0.324 & 0.235 & 0.140 & 0.148 & 0.174 & 0.000 & 0.025 & 0.331 & 0.384 & 0.361 & 0.295 & 0.391 & 0.258 & 0.360 & 0.382 & 0.127 & 0.116 & 0.304 & 0.205 \\
\hline $\mathrm{XMg}$ & 0.863 & 0.872 & 0.843 & 0.792 & 0.857 & 0.879 & 0.850 & 0.754 & 0.774 & 0.821 & 0.765 & 0.639 & 0.622 & 0.661 & 0.643 & 0.658 & 0.672 & 0.677 & 0.657 & 0.724 & 0.707 & 0.662 & 0.707 \\
\hline $\mathrm{XFe}^{3+}$ & 0.673 & 0.617 & 0.580 & 0.550 & 0.630 & 0.664 & 0.690 & 0.637 & 0.743 & 0.809 & 0.790 & 0.532 & 0.509 & 0.602 & 0.607 & 0.572 & 0.613 & 0.617 & 0.625 & 0.707 & 0.688 & 0.618 & 0.697 \\
\hline
\end{tabular}


Table 2 Chemical compositions of amphibole (continued).

\begin{tabular}{|c|c|c|c|c|c|c|c|c|c|c|c|c|c|c|c|c|c|c|c|c|c|c|c|}
\hline & & 59 & 59 & 9 & 9 & 59 & 159 & 159 & 9 & 3159 & 159 & 159 & 'B159 & 3159 & 3159 & B159 & B159 & $\mathrm{r}$ & B35 & YB35 & YB35 & YB35 & \\
\hline ock type & QS & QS & QS & QS & QS & QS & QS & QS & QS & QS & QS & $Q S$ & QS & os & QS & QS & QS & QS & BS & BS & BS & BS & $B S$ \\
\hline & 1 & 17 & 29 & & & & & & & & & & & & & & & & & & & & 2,2 \\
\hline \multirow[t]{3}{*}{ oint No. } & 4 & $46 \#$ & 93 & 96 & 97 & 98 & 99 & & & & & & & & & & & & & & & & 25 \\
\hline & & & core & core & core & core & re & & c & core & core & core & pre & le & le & mantle & mantle & mantle & core & core & ore & core & core \\
\hline & $\mathrm{Ba}$ & Wi & $\mathrm{Ba}$ & $\mathrm{Ba}$ & $\mathrm{Ba}$ & $\mathrm{Ba}$ & $\mathrm{Ba}$ & $3 a$ & $3 a$ & $\mathrm{Ba}$ & $\mathrm{Ba}$ & $\mathrm{Ba}$ & $\mathrm{Ba}$ & $\mathrm{Ba}$ & $3 a$ & $\mathrm{Ba}$ & $W_{1}$ & Wi & $\mathrm{Ba}$ & $\mathrm{Ba}$ & $\mathrm{Ba}$ & $\mathrm{Ba}$ & $\mathrm{Ba}$ \\
\hline $\mathrm{SiO}_{2}$ & 51.0 & 52.2 & 48.5 & 1 & 47 & .7 & .2 & .3 & 45.7 & 46.9 & 6.2 & 46.1 & 45.8 & 46.2 & 48.3 & 49.3 & 51.2 & 51.8 & 45.4 & 45.9 & 45 & 45.4 & 45.6 \\
\hline $\mathrm{TiO}_{2}$ & 11 & 04 & 19 & .21 & 19 & 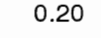 & 20 & 0.12 & .23 & 0.1 & 0.20 & 0.22 & 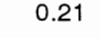 & 10 & 20 & 0.18 & 0.08 & 0.09 & 0.35 & 0.37 & 0.44 & 0.42 & 0.25 \\
\hline $\mathrm{Al}_{2} \mathrm{C}$ & & 51 & 1.0 & ).6 & 10.2 & .1 & 1.6 & .2 & .0 & 0.3 & 1.2 & 1.2 & .1 & & 12 & & & 98 & 6 & 1.8 & 11.6 & 11.8 & 10.8 \\
\hline $\mathrm{Cr}_{2}$ & & & & & & & & & & & & 0.01 & & & & 0.01 & 0.00 & 0 & 00 & 00 & 15 & 0.02 & 00 \\
\hline $\mathrm{Fe}$ & & & & & & & 2 & & & & 7.3 & 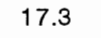 & 7.4 & 7.3 & 8.7 & 17.3 & & 7.5 & 17.2 & 7.2 & 17 & 17.5 & .3 \\
\hline $\mathrm{Mr}$ & & & & & & & & & & & & & & & & & & & & & & & 28 \\
\hline & & & & & & & & & & & & & & & & & & & & & & 12 & 9.82 \\
\hline & & & & & & & & & & & & & & & & & & & & & & & 22 \\
\hline & & & & & & & & & & & & & & & 33 & & & & & . & & & 63 \\
\hline 0 & & & & & 0.27 & & & & & & & 29 & & & 20 & 0.28 & & 12 & 42 & .48 & וד. & .55 & .39 \\
\hline & & & & & & & & & & & & & & & & & & & & & & & .3 \\
\hline & & & & & & & & & & & & & & & & & & & & 61 & & 6 & .60 \\
\hline & & & & & & & & & & & & & & & & & & & 3 & & & & .5 \\
\hline \multirow{2}{*}{ v total } & 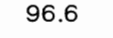 & & & & & & & & & & & & & & & & & & & & & & 7.1 \\
\hline & \multicolumn{23}{|c|}{ mic ratios } \\
\hline s & & & & & & & & & & & & & & & & & & & & & & & .747 \\
\hline & 41 & & 92 & 22 & 05 & 4 & & & & & & & & & & & & & 23 & 32 & 74 & 3 & 1.253 \\
\hline${ }^{[6]} \mathrm{Al}$ & & & & & & & & & & & & & & & & & & & & & & & 30 \\
\hline $\mathrm{Ti}$ & & & & & & & & & & & & & & & & & & & & & & & \\
\hline & & & & & & & & & & & & & & & & & & & .00 & .000 & & 0 & 0.000 \\
\hline & & & & 979 & 967 & 161 & 181 & 306 & 339 & 93 & 15 & 11 & 870 & 388 & 84 & 0.928 & 0.898 & 921 & 0.598 & 722 & 0.8 & 43 & .846 \\
\hline & & & & 023 & 094 & 931 & 22 & 56 & 291 & 312 & 6 & 0 & 278 & 265 & 166 & 190 & 8 & 7 & 1.518 & 57 & 1.311 & 93 & 295 \\
\hline & & & & 069 & 2 & & 58 & 059 & & & & 1 & 076 & & & & 77 & 7 & .0 & & & & 0.0 \\
\hline & & & & & 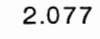 & & & 072 & & & & 036 & 062 & & & 292 & & 73 & .0 & 142 & & & 2.1 \\
\hline$c$ & 45 & 3 & 4 & 759 & 8 & 805 & 368 & 964 & 4 & & & & 079 & & & 10 & 2 & 6 & .379 & 21 & .304 & 43 & 03 \\
\hline$\Lambda$ & & & & & & & & & & & & & & & & & & & 24 & 0 & 1 & & 1.041 \\
\hline K & 017 & 0.026 & 0.043 & .048 & 0.051 & 0.045 & 0.050 & 0.058 & 061 & 054 & 1.060 & 055 & 062 & 061 & 552 & 52 & 030 & 022 & .079 & .089 & .095 & .102 & 0.074 \\
\hline & 15.184 & 15.148 & 15.428 & 15.447 & 15.429 & 15.387 & 15.281 & 15.572 & 15.551 & 15.518 & 15.474 & .541 & 15.554 & 389 & 15.350 & 15.303 & 15.221 & .166 & .581 & .520 & 15.520 & 15.535 & 15.418 \\
\hline${ }^{\left[{ }^{[B]}\right.} \mathrm{Na}$ & 1.055 & 1.247 & 1.176 & 1.241 & 1.202 & 1.195 & 1.132 & 1.036 & 0.996 & 37 & 1.015 & 930 & 921 & 15 & 35 & 90 & 1.128 & 094 & 621 & 679 & 696 & .657 & 0.697 \\
\hline 畐] $\mathrm{Na}$ & & 122 & 0.385 & & 0.378 & & & & & & & & & & & & & & & & & 433 & .344 \\
\hline & & 682 & 0.621 & 0.674 & 0.655 & 0.698 & 93 & & & & & 07 & & 18 & & & & 61 & 580 & 610 & 621 & .603 & 0.626 \\
\hline & 0.099 & .838 & 0.475 & 553 & . & 635 & 0 & 000 & 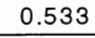 & 000 & 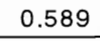 & . & . & 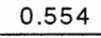 & 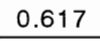 & . & I & 0.000 & .40 & 0.012 & $0.5 / 0$ & 0.531 & 0.573 \\
\hline
\end{tabular}


Table 2 Chemical compositions of amphibole (continued).

\begin{tabular}{|c|c|c|c|c|c|c|c|c|c|c|c|c|c|c|c|c|c|c|c|c|c|c|c|}
\hline Sample No. & YB35 & YB35 & YB35 & YB35 & YB35 & YB35 & YB35 & YB35 & YB35 & YB35 & YB35 & YB35 & YB35 & YB35 & YB35 & YB35 & YB35 & YB35 & YB35 & YB35 & YB35 & YB35 & YB35 \\
\hline Rock type & BS & BS & BS & BS & BS & BS & BS & BS & BS & BS & BS & BS & BS & BS & BS & BS & BS & BS & BS & BS & BS & BS & BS \\
\hline Grain No. & 2,2 & 2,2 & 2,2 & 2,2 & 2,2 & 2,2 & 2,2 & 3,3 & 3,3 & 3,3 & 3,3 & 3,3 & 3,3 & 3,3 & 3,3 & 3,3 & 3,3 & 7,5 & 7,5 & 7,5 & 7,5 & 7,5 & 7,5 \\
\hline \multirow[t]{3}{*}{ Point No. } & 26 & 27 & 28 & 29 & 30 & 31 & 32\# & 43 & 44 & 45 & 46 & 47 & 48 & $49 \#$ & 51 & 52 & 53 & 67 & 68 & 70 & 71 & 72 & 73 \\
\hline & core & core & mantle & mantle & mantle & mantle & mantle & core & core & core & core & core & core & mantle & mantle & mantle & mantle & core & core & core & mantle & mantle & mantle \\
\hline & $\mathrm{Ba}$ & $\mathrm{Ba}$ & Wi & Wi & Wi & Wi & Mrb & $\mathrm{Ba}$ & $\mathrm{Ba}$ & $\mathrm{Ba}$ & $\mathrm{Ba}$ & $\mathrm{Ba}$ & $\mathrm{Ba}$ & Wi & Wi & Wi & Wi & $\mathrm{Ba}$ & $\mathrm{Ba}$ & $\mathrm{Ba}$ & Wi & Wi & Wi \\
\hline $\mathrm{SiO}_{2}$ & 45.5 & 46.1 & 52.1 & 53.5 & 53.9 & 54.6 & 54.6 & 46.3 & 46.3 & 45.5 & 46.7 & 46.6 & 45.7 & 52.1 & 51.3 & 53.4 & 53.7 & 45.8 & 46.0 & 45.9 & 53.0 & 53.6 & 53.6 \\
\hline $\mathrm{TiO}_{2}$ & 0.29 & 0.34 & 0.13 & 0.07 & 0.04 & 0.04 & 0.05 & 0.30 & 0.31 & 0.19 & 0.33 & 0.22 & 0.22 & 0.06 & 0.06 & 0.03 & 0.06 & 0.38 & 0.33 & 0.33 & 0.10 & 0.05 & 0.03 \\
\hline $\mathrm{Al}_{2} \mathrm{O}_{3}$ & 11.2 & 10.7 & 4.83 & 3.30 & 3.36 & 3.46 & 3.84 & 10.5 & 10.6 & 11.3 & 10.2 & 10.3 & 10.9 & 3.98 & 3.74 & 2.04 & 1.74 & 10.9 & 10.9 & 11.2 & 3.88 & 3.34 & 3.25 \\
\hline $\mathrm{Cr}_{2} \mathrm{O}_{3}$ & 0.00 & 0.00 & 0.06 & 0.00 & 0.00 & 0.00 & 0.00 & 0.05 & 0.00 & 0.02 & 0.01 & 0.01 & 0.00 & 0.00 & 0.02 & 0.05 & 0.00 & 0.00 & 0.00 & 0.00 & 0.00 & 0.00 & 0.02 \\
\hline $\mathrm{FeO}^{*}$ & 17.4 & 17.4 & 19.1 & 18.7 & 20.2 & 20.9 & 21.2 & 16.6 & 16.9 & 18.0 & 17.6 & 17.6 & 17.6 & 20.0 & 20.0 & 19.4 & 18.7 & 17.9 & 17.3 & 18.2 & 19.9 & 21.0 & 21.7 \\
\hline $\mathrm{MnO}$ & .37 & 24 & 22 & 0.16 & 0.11 & 0.15 & 0.10 & 0.32 & 0.35 & 0.35 & 0.35 & 0.42 & 0.29 & 0.18 & 0.26 & 0.21 & 0.21 & 0.28 & 0.30 & 0.23 & 0.17 & 0.25 & 0.14 \\
\hline $\mathrm{MgO}$ & 9.53 & 9.83 & 10.3 & 10.5 & 10.2 & 9.39 & 9.30 & 10.3 & 9.90 & 9.44 & 9.86 & 9.70 & 9.42 & 9.76 & 10.4 & 11.5 & 11.7 & 9.48 & 9.85 & 9.66 & 10.3 & 9.00 & 9.08 \\
\hline $\mathrm{CaO}$ & 8.40 & 8.34 & 6.35 & 6.12 & 4.76 & 4.08 & 3.02 & 8.42 & 7.92 & 8.14 & 8.20 & 7.83 & 8.17 & 6.17 & 8.76 & 8.94 & 9.54 & 8.72 & 8.59 & 8.29 & 5.06 & 4.03 & 4.01 \\
\hline $\mathrm{Na}_{2} \mathrm{O}$ & 3.85 & 4.08 & 4.08 & 4.22 & 4.92 & 5.27 & 5.52 & 3.48 & 3.72 & 3.76 & 3.98 & 3.84 & 4.19 & 3.97 & 2.44 & 2.47 & 2.20 & 3.92 & 3.97 & 4.11 & 4.45 & 4.85 & 5.08 \\
\hline $\mathrm{K}_{2} \mathrm{O}$ & 0.39 & 0.37 & 0.18 & 0.13 & 0.10 & 0.04 & 0.08 & 0.34 & 0.31 & 0.36 & 0.29 & 0.28 & 0.32 & 0.15 & 0.32 & 0.12 & 0.10 & 0.48 & 0.49 & 0.43 & 0.08 & 0.09 & 0.08 \\
\hline Total & 96.9 & 97.4 & 97.4 & 96.7 & 97.6 & 97.9 & 97.7 & 96.6 & 96.3 & 97.1 & 97.5 & 96.8 & 96.8 & 96.4 & 97.3 & 98.2 & 98.0 & 97.9 & 97.7 & 98.4 & 96.9 & 96.2 & 97.0 \\
\hline $\mathrm{Fe}_{2} \mathrm{O}_{3}$ & 6.34 & 5.83 & 7.62 & 5.67 & 9.17 & 8.76 & 11.5 & 7.16 & 7.41 & 8.22 & 6.47 & 7.73 & 5.76 & 7.58 & 6.24 & 5.54 & 3.50 & 5.16 & 5.43 & 7.17 & 10.4 & 9.56 & 10.2 \\
\hline $\mathrm{FeO}$ & 11.7 & 12.2 & 12.2 & 13.6 & 12.0 & 13.0 & 10.8 & 10.2 & 10.2 & 10.6 & 11.8 & 10.6 & 12.4 & 13.2 & 14.4 & 14.4 & 15.6 & 13.3 & 12.4 & 11.7 & 10.5 & 12.4 & 12.5 \\
\hline \multirow[t]{2}{*}{ New total } & 97.6 & 98.0 & 98.1 & 97.3 & 98.6 & 98.8 & 98.8 & 97.4 & 97.0 & 97.9 & 98.2 & 97.5 & 97.4 & 97.2 & 97.9 & 98.7 & 98.4 & 98.4 & 98.3 & 99.0 & 97.9 & 97.2 & 98.0 \\
\hline & \multicolumn{23}{|c|}{ Atomic ratios $(\mathrm{O}=23)$} \\
\hline $\mathrm{Si}$ & 6.722 & 6.781 & 7.572 & 7.826 & 7.778 & 7.859 & 7.814 & 6.805 & 6.823 & 6.691 & 6.845 & 6.849 & 6.773 & 7.674 & 7.560 & 7.764 & 7.839 & 6.745 & 6.757 & 6.692 & 7.673 & 7.850 & 7.805 \\
\hline${ }^{[4]} \mathrm{Al}$ & 1.278 & 1.219 & 0.428 & 0.174 & 0.222 & 0.141 & 0.186 & 1.195 & 1.177 & 1.309 & 1.155 & 1.151 & 1.227 & 0.326 & 0.440 & 0.236 & 0.161 & 1.255 & 1.243 & 1.308 & .327 & 150 & 0.195 \\
\hline${ }^{[6]} \mathrm{Al}$ & 0.673 & 0.636 & 0.399 & 0.395 & 0.350 & 0.446 & 0.461 & 0.624 & 0.664 & 0.650 & 0.607 & 0.633 & 0.677 & 0.364 & 0.209 & 0.114 & 0.138 & 0. & 0.644 & 0.617 & 36 & 426 & 0.363 \\
\hline $\mathrm{Ti}$ & 0.032 & 0.038 & 0.014 & 0.008 & 0.004 & 0.004 & 0.005 & 0.033 & 0.034 & 0.021 & 0.036 & 0.024 & .025 & 0.007 & 0.007 & 0.003 & 0.007 & 0.042 & 0.036 & 0.036 & 11 & 006 & 03 \\
\hline $\mathrm{Cr}$ & 0.000 & 0.000 & 0.007 & 0.000 & 0.000 & 0.000 & 0.000 & 0.006 & 0.000 & 0.002 & 0.001 & 0.001 & 0.000 & 0.000 & 0.002 & 0.006 & 0.000 & 0.000 & 0.000 & 0.000 & 0.000 & 0.000 & 0.002 \\
\hline $\mathrm{Fe}^{3+}$ & 0.705 & 0.645 & 0.834 & 0.624 & 0.996 & 0.949 & 1.242 & 0.792 & 0.822 & 0.910 & 0.713 & 0.855 & 0.643 & 0.840 & 0.692 & 0.606 & 0.384 & 0.572 & 0.600 & 0.787 & 1.135 & 1.054 & 1.122 \\
\hline $\mathrm{Fe}^{2+}$ & 1.445 & 1.495 & 1.488 & 1.664 & 1.442 & 1.567 & 1.295 & 1.249 & 1.261 & 1.304 & 1.444 & 1.309 & 1.539 & 1.624 & 1.773 & 1.753 & 1.899 & 1.632 & 1.525 & 1.432 & 1.274 & 1.518 & 1.521 \\
\hline $\mathrm{Mn}$ & 0.046 & 0.030 & 0.027 & 0.020 & 0.013 & 0.018 & 0.012 & 0.040 & 0.044 & 0.044 & 0.043 & 0.052 & 0.036 & 0.022 & 0.032 & 0.026 & 0.026 & 0.035 & 0.037 & 0.028 & 0.021 & 0.031 & 0.017 \\
\hline $\mathrm{Mg}$ & 2.099 & 2.156 & 2.232 & 2.290 & 2.194 & 2.015 & 1.984 & 2.257 & 2.175 & 2.070 & 2.155 & 2.125 & 2.081 & 2.143 & 2.285 & 2.493 & 2.546 & 2.081 & 2.157 & 2.100 & 2.223 & 1.965 & 1.971 \\
\hline $\mathrm{Ca}$ & 1.330 & 1.314 & 0.989 & 0.959 & 0.736 & 0.629 & 0.463 & 1.326 & 1.251 & 1.283 & 1.288 & 1.233 & 1.297 & 0.974 & 1.383 & 1.393 & 1.492 & 1.376 & 1.352 & 1.295 & 0.785 & 0.632 & 0.626 \\
\hline $\mathrm{Na}$ & 1.103 & 1.164 & 1.150 & 1.197 & 1.377 & 1.471 & 1.532 & 0.992 & 1.063 & 1.072 & 1.131 & 1.094 & 1.204 & 1.134 & 0.697 & 0.696 & 0.623 & 1.119 & 1.131 & 1.162 & 1.249 & 1.377 & 1.434 \\
\hline $\mathrm{K}$ & 0.074 & 0.069 & 0.033 & 0.024 & 0.018 & 0.007 & 0.015 & 0.064 & 0.058 & 0.068 & 0.054 & 0.053 & 0.061 & 0.028 & 0.060 & 0.022 & 0.019 & 0.090 & 0.092 & 0.080 & 0.015 & 0.017 & 0.015 \\
\hline Total & 15.506 & 15.548 & 15.172 & 5.180 & 15.131 & 15.107 & 15.009 & 15.381 & 15.372 & 15.422 & 15.473 & 15.380 & 15.562 & 15.136 & 15.140 & 15.111 & 15.133 & 15.585 & 15.574 & 15.537 & 15.049 & 15.026 & 15.075 \\
\hline${ }^{[\mathrm{B}]} \mathrm{Na}$ & 0.670 & 0.686 & 1.011 & 1.041 & 1.264 & 1.371 & 1.532 & 0.674 & 0.749 & 0.717 & 0.712 & 0.767 & 0.703 & 1.026 & 0.617 & 0.607 & 0.508 & 0.624 & 0.648 & 0.705 & 1.215 & 1.368 & 1.374 \\
\hline${ }^{[\mathrm{A}]} \mathrm{Na}$ & 0.433 & 0.478 & 0.138 & 0.156 & 0.113 & 0.100 & 0.000 & 0.318 & 0.313 & 0.355 & 0.419 & 0.327 & 0.501 & 0.107 & 0.080 & 0.089 & 0.115 & 0.495 & 0.483 & 0.457 & .034 & 0.010 & 0.060 \\
\hline$X M g$ & 0.592 & 0.590 & 0.600 & 0.579 & 0.603 & 0.563 & 0.605 & 0.644 & 0.633 & 0.613 & 0.599 & 0.619 & 0.575 & 0.569 & 0.563 & 0.587 & 0.573 & 0.560 & 0.586 & 0.595 & 0.636 & 0.564 & 0.565 \\
\hline $\mathrm{XFe}^{3+}$ & 0.512 & 0.503 & 0.676 & 0.612 & 0.740 & 0.680 & 0.729 & 0.559 & 0.553 & 0.583 & 0.540 & 0.574 & 0.487 & 0.697 & 0.768 & 0.842 & 0.735 & 0.473 & 0.482 & 0.561 & 0.772 & 0.712 & 0.755 \\
\hline
\end{tabular}


Table 2 Chemical compositions of amphibole (continued).

\begin{tabular}{|c|c|c|c|c|c|c|c|c|c|c|c|c|c|c|c|c|c|c|c|c|c|c|c|}
\hline Sample No. & YB35 & YB35 & YB46 & YB46 & YB46 & YB46 & YB46 & YB46 & YB46 & YB46 & YB46 & YB46 & YB46 & YB46 & YB46 & YB46 & YB46 & YB46 & YB46 & YB46 & YB46 & YB46 & YB46 \\
\hline Rock type & BS & BS & BS & BS & BS & BS & BS & BS & BS & BS & BS & BS & BS & BS & BS & BS & BS & BS & BS & BS & BS & BS & BS \\
\hline Grain No. & 7,5 & 7,5 & 13,2 & 13,2 & 13,2 & 13,2 & 13,2 & 13,2 & 13,2 & 13,2 & 13,2 & 13,2 & 13,2 & 13,2 & 13,2 & 13,2 & 13,2 & 13,2 & 13,2 & 13,2 & 13,2 & 13,2 & 18,3 \\
\hline \multirow[t]{3}{*}{ Point No. } & $74 \#$ & 75 & 42 & 43 & 44 & 45 & 46 & 47 & 48 & 49 & 50 & $51 \#$ & 52 & 53 & 54 & 55 & 56 & 57 & 58 & 59 & 60 & 61 & 83 \\
\hline & mantle & mantle & core & core & core & core & core & core & core & mantle & mantle & mantle & mantle & mantle & mantle & mantle & mantle & mantle & mantle & mantle & mantle & mantle & core \\
\hline & Wi & $W_{i}$ & $\mathrm{Ba}$ & $\mathrm{Ba}$ & $\mathrm{Ba}$ & $\mathrm{Ba}$ & $\mathrm{Ba}$ & $\mathrm{Ba}$ & $\mathrm{Ba}$ & $\mathrm{Ba}$ & Mrb & Mrb & Wi & Wi & Wi & Wi & $W_{i}$ & Wi & Act & Act & Act & Act & $\mathrm{Ba}$ \\
\hline $\mathrm{SiO}_{2}$ & 53.8 & 54.0 & 46.6 & 46.7 & 47.4 & 47.5 & 47.2 & 46.5 & 46.2 & 48.4 & 54.4 & 56.4 & 55.5 & 54.3 & 54.9 & 55.3 & 54.7 & 53.8 & 55.3 & 55.4 & 55.2 & 54.2 & 47.1 \\
\hline $\mathrm{TiO}_{2}$ & 0.04 & 0.03 & 0.46 & 0.48 & 0.37 & 0.42 & 0.42 & 0.41 & 0.35 & 0.19 & 0.11 & 0.04 & 0.07 & 0.11 & 0.04 & 0.06 & 0.09 & 0.01 & 0.00 & 0.01 & 0.00 & 0.05 & 0.37 \\
\hline $\mathrm{Al}_{2} \mathrm{O}_{3}$ & 3.46 & 3.17 & 10.4 & 9.95 & 9.88 & 9.84 & 9.88 & 9.84 & 10.0 & 7.94 & 6.59 & 3.47 & 2.77 & 2.31 & 2.48 & 2.73 & 2.18 & 2.94 & 1.72 & 1.81 & 0.84 & 2.02 & 9.71 \\
\hline $\mathrm{Cr}_{2} \mathrm{O}_{3}$ & 0.03 & 0.00 & 0.00 & 0.03 & 0.00 & 0.02 & 0.00 & 0.03 & 0.03 & 0.03 & 0.00 & 0.00 & 0.00 & 0.01 & 0.00 & 0.00 & 0.04 & 0.00 & 0.00 & 0.03 & 0.06 & 0.00 & 0.03 \\
\hline $\mathrm{FeO}^{*}$ & 21.9 & 19.8 & 15.8 & 15.5 & 15.2 & 16.2 & 16.0 & 15.5 & 16.1 & 17.1 & 16.7 & 18.2 & 15.8 & 13.5 & 13.5 & 14.1 & 12.7 & 13.7 & 11.5 & 12.6 & 12.5 & 12.8 & 14.2 \\
\hline $\mathrm{MnO}$ & 0.14 & 0.24 & 0.00 & 0.05 & 0.11 & 0.08 & 0.04 & 0.11 & 0.05 & 0.06 & 0.08 & 0.05 & 0.10 & 0.18 & 0.18 & 0.16 & 0.16 & 0.23 & 0.21 & 0.23 & 0.26 & 0.19 & 0.22 \\
\hline $\mathrm{MgO}$ & 8.74 & 9.96 & 11.3 & 11.0 & 11.1 & 11.3 & 11.3 & 11.3 & 10.8 & 11.1 & 10.7 & 10.9 & 12.9 & 15.1 & 14.5 & 14.6 & 15.2 & 14.8 & 16.5 & 16.7 & 15.9 & 15.4 & 11.7 \\
\hline $\mathrm{CaO}$ & 3.56 & 5.66 & 7.74 & 7.74 & 7.82 & 7.85 & 7.93 & 8.40 & 8.37 & 7.68 & 2.90 & 1.76 & 4.30 & 8.46 & 8.00 & 7.83 & 9.68 & 9.56 & 10.4 & 10.3 & 10.2 & 9.78 & 8.48 \\
\hline $\mathrm{Na}_{2} \mathrm{O}$ & .57 & 4.40 & 4.31 & 4.24 & 4.39 & 4.41 & 4.16 & 4.01 & 4.01 & 3.57 & 6.05 & 6.32 & 4.95 & 2.82 & 3.25 & 3.00 & 2.18 & 2.40 & 1.66 & 1.63 & 1.67 & 1.98 & 3.86 \\
\hline $\mathrm{K}_{2} \mathrm{O}$ & 0.08 & 0.12 & 0.57 & 0.51 & 0.47 & 0.41 & 0.48 & 0.55 & 0.57 & 0.32 & 0.08 & 0.04 & 0.04 & 0.07 & 0.07 & 0.05 & 0.12 & 0.11 & 0.06 & 0.08 & 0.07 & 0.07 & 0.50 \\
\hline Total & 97.3 & 97.4 & 97.2 & 96.2 & 96.7 & 98.0 & 97.4 & 96.7 & 96.5 & 96.4 & 97.6 & 97.2 & 96.4 & 96.9 & 96.9 & 97.8 & 97.1 & 97.6 & 97.4 & 98.8 & 96.7 & 96.5 & 96.2 \\
\hline $\mathrm{Fe}_{2} \mathrm{O}_{3}$ & 9.62 & 6.51 & 7.32 & 5.83 & 4.72 & 6.85 & 6.94 & 5.55 & 5.35 & 8.50 & 9.46 & 11.4 & 9.70 & 6.54 & 4.71 & 7.13 & 3.20 & 4.81 & 3.85 & 6.73 & 3.70 & 4.52 & 4.53 \\
\hline $\mathrm{FeO}$ & 13.2 & 13.9 & 9.21 & 10.3 & 11.0 & 10.0 & 9.75 & 10.5 & 11.3 & 9.45 & 8.19 & 7.96 & 7.07 & 7.62 & 9.26 & 7.69 & 9.82 & 9.37 & 8.03 & 6.54 & 9.17 & 8.73 & 10.1 \\
\hline \multirow[t]{2}{*}{ New total } & 98.2 & 98.0 & 97.9 & 96.8 & 97.3 & 98.7 & 98.1 & 97.2 & 97.0 & 97.2 & 98.6 & 98.3 & 97.4 & 97.5 & 97.4 & 98.6 & 97.4 & 98.0 & 97.7 & 99.5 & 97.1 & 96.9 & 96.6 \\
\hline & \multicolumn{23}{|c|}{ Atomic ratios $(\mathrm{O}=23)$} \\
\hline $\mathrm{Si}$ & 7.824 & 7.855 & 6.787 & 6.882 & 6.949 & 6.869 & 6.862 & 6.844 & 6.834 & 7.084 & 7.667 & 7.967 & 7.898 & 7.751 & 7.850 & 7.792 & 7.833 & 7.687 & 7.838 & 7.728 & 7.919 & 7.791 & 6.930 \\
\hline${ }^{[4]} \mathrm{Al}$ & 0.176 & 0.145 & 1.213 & 1.118 & 1.051 & 1.131 & 1.138 & 1.156 & 1.166 & 0.916 & 0.333 & 0.033 & 0.102 & 0.249 & 0.150 & 0.208 & 0.167 & 0.313 & 0.162 & 0.272 & 0.081 & 0.209 & 1.070 \\
\hline${ }^{[6]} \mathrm{Al}$ & 0.417 & 0.399 & 0.572 & 0.610 & 0.656 & 0.547 & 0.555 & 0.550 & 0.578 & 0.453 & 0.762 & 0.544 & 0.363 & 0.140 & 0.268 & 0.246 & 0.201 & 0.182 & 0.125 & 0.026 & 0.061 & 0.133 & 0.614 \\
\hline $\mathrm{Ti}$ & 0.004 & 0.003 & 0.050 & 0.053 & 0.041 & 0.046 & 0.046 & 0.045 & 0.039 & 0.021 & 0.012 & 0.004 & 0.007 & 0.012 & 0.004 & 0.006 & 0.010 & 0.001 & 0.000 & 0.001 & 0.000 & 0.005 & 0.041 \\
\hline $\mathrm{Cr}$ & 0.003 & 0.000 & 0.000 & 0.003 & 0.000 & 0.002 & 0.000 & 0.003 & 0.004 & 0.003 & 0.000 & 0.000 & 0.000 & 0.001 & 0.000 & 0.000 & 0.005 & 0.000 & 0.000 & 0.003 & 0.007 & 0.000 & 0.003 \\
\hline $\mathrm{Fe}^{3+}$ & 1.053 & 0.712 & 0.802 & 0.646 & 0.521 & 0.745 & 0.759 & 0.615 & 0.596 & 0.936 & 1.004 & 1.209 & 1.039 & 0.702 & 0.507 & 0.756 & 0.345 & 0.518 & 0.411 & 0.707 & 0.400 & 0.489 & 0.501 \\
\hline $\mathrm{Fe}^{2+}$ & 1.611 & 1.696 & 1.122 & 1.264 & 1.343 & 1.214 & 1.186 & 1.293 & 1.396 & 1.157 & 0.965 & 0.941 & 0.841 & 0.909 & 1.107 & 0.906 & 1.176 & 1.119 & 0.952 & 0.763 & 1.100 & 1.050 & 1.246 \\
\hline $\mathrm{Mn}$ & 0.017 & 0.030 & 0.000 & 0.006 & 0.014 & 0.010 & 0.005 & 0.014 & 0.006 & 0.007 & 0.010 & 0.006 & 0.012 & 0.022 & 0.022 & 0.019 & 0.019 & 0.028 & 0.025 & 0.027 & 0.032 & 0.023 & 0.027 \\
\hline $\mathrm{Mg}$ & 1.895 & 2.160 & 2.453 & 2.417 & 2.426 & 2.436 & 2.449 & 2.479 & 2.382 & 2.422 & 2.248 & 2.295 & 2.737 & 3.214 & 3.091 & 3.067 & 3.245 & 3.152 & 3.486 & 3.473 & 3.401 & 3.300 & 2.566 \\
\hline $\mathrm{Ca}$ & 0.555 & 0.882 & 1.208 & 1.222 & 1.228 & 1.216 & 1.235 & 1.325 & 1.327 & 1.204 & 0.438 & 0.266 & 0.656 & 1.294 & 1.226 & 1.182 & 1.485 & 1.463 & 1.579 & 1.539 & 1.568 & 1.506 & 1.337 \\
\hline $\mathrm{Na}$ & 1.571 & 1.241 & 1.217 & 1.212 & 1.248 & 1.237 & 1.173 & 1.144 & 1.150 & 1.013 & 1.653 & 1.731 & 1.366 & 0.781 & 0.901 & 0.820 & 0.605 & 0.665 & 0.456 & 0.441 & 0.465 & 0.552 & 1.101 \\
\hline $\mathrm{K}$ & 0.015 & 0.022 & 0.106 & 0.096 & 0.088 & 0.076 & 0.089 & 0.103 & 0.108 & 0.060 & 0.014 & 0.007 & 0.007 & 0.013 & 0.013 & 0.009 & 0.022 & 0.020 & 0.011 & 0.014 & 0.013 & 0.013 & 0.094 \\
\hline Total & 15.140 & 15.145 & 15.531 & 15.529 & 15.564 & 15.529 & 15.497 & 15.572 & 15.584 & 15.277 & 15.106 & 15.004 & 15.029 & 15.087 & 15.140 & 15.011 & 15.112 & 15.148 & 15.046 & 14.995 & 15.045 & 15.071 & 15.532 \\
\hline [B] $\mathrm{Na}$ & 1.445 & 1.118 & 0.792 & 0.778 & 0.772 & 0.784 & 0.765 & 0.675 & 0.673 & 0.796 & 1.562 & 1.731 & 1.344 & 0.706 & 0.774 & 0.818 & 0.515 & 0.537 & 0.421 & 0.441 & 0.432 & 0.494 & 0.663 \\
\hline${ }^{[\mathrm{A}]} \mathrm{Na}$ & 0.125 & 0.123 & 0.425 & 0.434 & 0.476 & 0.453 & 0.408 & 0.469 & 0.477 & 0.217 & 0.091 & 0.000 & 0.022 & 0.074 & 0.127 & 0.002 & 0.090 & 0.128 & 0.036 & 0.000 & 0.032 & 0.058 & 0.438 \\
\hline XMg & 0.541 & 0.560 & 0.686 & 0.657 & 0.644 & 0.667 & 0.674 & 0.657 & 0.630 & 0.677 & 0.700 & 0.709 & 0.765 & 0.779 & 0.736 & 0.772 & 0.734 & 0.738 & 0.786 & 0.820 & 0.756 & 0.759 & 0.673 \\
\hline $\mathrm{XFe}^{3+}$ & 0.716 & 0.641 & 0.584 & 0.514 & 0.443 & 0.577 & 0.578 & 0.528 & 0.508 & 0.674 & 0.568 & 0.690 & 0.741 & 0.834 & 0.654 & 0.755 & 0.632 & 0.740 & 0.766 & 0.965 & 0.867 & 0.786 & 0.449 \\
\hline
\end{tabular}


Table 2 Chemical compositions of amphibole (continued).

\begin{tabular}{|c|c|c|c|c|c|c|c|c|c|c|c|c|c|c|}
\hline Sample No. & YB46 & YB46 & YB46 & YB46 & YB46 & YB46 & YB46 & YB46 & YB46 & YB46 & YB46 & $\begin{array}{l}\text { YB46 } \\
\end{array}$ & YB46 & YB46 \\
\hline Rock type & BS & BS & BS & BS & BS & BS & BS & BS & BS & BS & BS & BS & BS & BS \\
\hline Grain No. & 18,3 & 18,3 & 18,3 & 18,3 & 18,3 & 18,3 & 18,3 & 18,3 & 18,3 & 18,3 & 18,3 & 18,3 & 18,3 & 18,3 \\
\hline \multirow[t]{3}{*}{ Point No. } & 84 & 85 & 86 & 87 & 88 & 89 & $90 \#$ & 91 & 92 & 93 & 94 & 95 & 96 & 97 \\
\hline & core & core & core & core & core & mantle & mantle & mantle & mantle & mantle & mantle & mantle & mantle & mantle \\
\hline & $\mathrm{Ba}$ & $\mathrm{Ba}$ & $\mathrm{Ba}$ & $\mathrm{Ba}$ & $\mathrm{Ba}$ & $\mathrm{Wi}$ & Wi & Wi & Wi & Wi & Wi & Act & Act & Act \\
\hline$\overline{\mathrm{SiO}_{2}}$ & 47.1 & 47.2 & 47.3 & 47.4 & 47.3 & 55.9 & 55.9 & 55.9 & 55.9 & 54.6 & 54.0 & 55.0 & 56.1 & 56.4 \\
\hline $\mathrm{TiO}_{2}$ & 0.37 & 0.38 & 0.39 & 0.47 & 0.40 & 0.05 & 0.03 & 0.04 & 0.03 & 0.00 & 0.01 & 0.01 & 0.02 & 0.05 \\
\hline $\mathrm{Al}_{2} \mathrm{O}_{3}$ & 9.60 & 9.58 & 9.71 & 9.48 & 9.82 & 2.26 & 2.62 & 2.62 & 2.31 & 2.58 & 1.85 & 1.65 & 0.77 & 0.69 \\
\hline $\mathrm{Cr}_{2} \mathrm{O}_{3}$ & 0.00 & 0.02 & 0.00 & 0.00 & 0.05 & 0.00 & 0.00 & 0.00 & 0.04 & 0.07 & 0.01 & 0.00 & 0.00 & 0.01 \\
\hline $\mathrm{FeO}^{*}$ & 15.0 & 16.0 & 16.0 & 15.4 & 15.4 & 16.5 & 16.4 & 15.5 & 15.4 & 14.5 & 13.2 & 13.8 & 12.6 & 12.9 \\
\hline $\mathrm{MnO}$ & 0.19 & 0.18 & 0.19 & 0.10 & 0.10 & 0.21 & 0.18 & 0.20 & 0.18 & 0.26 & 0.29 & 0.31 & 0.30 & 0.36 \\
\hline $\mathrm{MgO}$ & 12.0 & 11.4 & 11.4 & 11.7 & 11.5 & 12.9 & 13.0 & 13.1 & 13.4 & 14.2 & 14.9 & 15.4 & 16.6 & 15.6 \\
\hline $\mathrm{CaO}$ & 8.37 & 7.82 & 8.16 & 8.51 & 8.53 & 4.46 & 3.68 & 4.41 & 4.79 & 8.13 & 9.16 & 9.82 & 11.0 & 9.93 \\
\hline $\mathrm{Na}_{2} \mathrm{O}$ & 3.88 & 3.88 & 3.87 & 3.88 & 3.97 & 4.79 & 5.36 & 4.85 & 4.74 & 3.13 & 2.20 & 1.68 & 1.33 & 1.46 \\
\hline $\mathrm{K}_{2} \mathrm{O}$ & 0.47 & 0.55 & 0.43 & 0.55 & 0.55 & 0.03 & 0.06 & 0.00 & 0.04 & 0.06 & 0.09 & 0.08 & 0.06 & 0.06 \\
\hline Total & 97.0 & 97.0 & 97.5 & 97.5 & 97.6 & 97.1 & 97.2 & 96.6 & 96.8 & 97.5 & 95.7 & 97.8 & 98.8 & 97.5 \\
\hline $\mathrm{Fe}_{2} \mathrm{O}_{3}$ & 6.96 & 8.18 & 7.49 & 5.71 & 5.22 & 10.4 & 11.5 & 9.63 & 9.23 & 5.93 & 5.02 & 6.29 & 4.04 & 4.06 \\
\hline $\mathrm{FeO}$ & 8.74 & 8.64 & 9.26 & 10.3 & 10.7 & 7.15 & 6.06 & 6.84 & 7.09 & 9.16 & 8.68 & 8.14 & 8.97 & 9.25 \\
\hline \multirow[t]{2}{*}{ New total } & 97.7 & 97.8 & 98.2 & 98.1 & 98.1 & 98.2 & 98.4 & 97.6 & 97.8 & 98.1 & 96.2 & 98.4 & 99.2 & 97.9 \\
\hline & \multicolumn{14}{|c|}{ Atomic ratios $(\mathrm{O}=23)$} \\
\hline $\mathrm{Si}$ & 6.858 & 6.870 & 6.865 & 6.898 & 6.884 & 7.915 & 7.877 & 7.927 & 7.926 & 7.782 & 7.827 & 7.796 & 7.879 & 8.004 \\
\hline${ }^{[4]} \mathrm{Al}$ & 1.142 & 1.130 & 1.135 & 1.102 & 1.116 & 0.085 & 0.123 & 0.073 & 0.074 & 0.218 & 0.173 & 0.204 & 0.121 & 0.000 \\
\hline${ }^{[6]} \mathrm{Al}$ & 0.505 & 0.513 & 0.526 & 0.524 & 0.569 & 0.292 & 0.312 & 0.364 & 0.312 & 0.215 & 0.143 & 0.072 & 0.007 & 0.115 \\
\hline $\mathrm{Ti}$ & 0.041 & 0.042 & 0.043 & 0.051 & 0.044 & 0.005 & 0.003 & 0.004 & 0.003 & 0.000 & 0.001 & 0.001 & 0.002 & 0.005 \\
\hline $\mathrm{Cr}$ & 0.000 & 0.002 & 0.000 & 0.000 & 0.006 & 0.000 & 0.000 & 0.000 & 0.004 & 0.008 & 0.001 & 0.000 & 0.000 & 0.001 \\
\hline $\mathrm{Fe}^{3+}$ & 0.762 & 0.896 & 0.818 & 0.625 & 0.571 & 1.108 & 1.219 & 1.027 & 0.985 & 0.636 & 0.548 & 0.671 & 0.427 & 0.433 \\
\hline $\mathrm{Fe}^{2+}$ & 1.064 & 1.051 & 1.124 & 1.249 & 1.303 & 0.846 & 0.714 & 0.811 & 0.841 & 1.092 & 1.052 & 0.965 & 1.053 & 1.098 \\
\hline $\mathrm{Mn}$ & 0.023 & 0.022 & 0.023 & 0.012 & 0.012 & 0.025 & 0.021 & 0.024 & 0.022 & 0.031 & 0.036 & 0.037 & 0.036 & 0.043 \\
\hline $\mathrm{Mg}$ & 2.605 & 2.474 & 2.467 & 2.538 & 2.495 & 2.723 & 2.731 & 2.769 & 2.832 & 3.017 & 3.219 & 3.254 & 3.476 & 3.300 \\
\hline $\mathrm{Ca}$ & 1.306 & 1.219 & 1.269 & 1.327 & 1.330 & 0.677 & 0.556 & 0.670 & 0.728 & 1.242 & 1.422 & 1.491 & 1.655 & 1.510 \\
\hline $\mathrm{Na}$ & 1.095 & 1.095 & 1.089 & 1.095 & 1.120 & 1.315 & 1.464 & 1.333 & 1.303 & 0.865 & 0.618 & 0.462 & 0.362 & 0.402 \\
\hline $\mathrm{K}$ & 0.087 & 0.102 & 0.080 & 0.102 & 0.102 & 0.005 & 0.011 & 0.000 & 0.007 & 0.011 & 0.017 & 0.014 & 0.011 & 0.011 \\
\hline Total & 15.488 & 15.416 & 15.437 & 15.524 & 15.553 & 14.997 & 15.031 & 15.003 & 15.038 & 15.117 & 15.057 & 14.968 & 15.028 & 14.922 \\
\hline${ }^{[B]} \mathrm{Na}$ & 0.694 & 0.781 & 0.731 & 0.673 & 0.670 & 1.315 & 1.444 & 1.330 & 1.272 & 0.758 & 0.578 & 0.462 & 0.345 & 0.402 \\
\hline${ }^{[\mathrm{A}]} \mathrm{Na}$ & 0.401 & 0.314 & 0.358 & 0.422 & 0.450 & 0.000 & 0.020 & 0.003 & 0.031 & 0.106 & 0.041 & 0.000 & 0.017 & 0.000 \\
\hline $\mathrm{XMg}$ & 0.710 & 0.702 & 0.687 & 0.670 & 0.657 & 0.763 & 0.793 & 0.773 & 0.771 & 0.734 & 0.754 & 0.771 & 0.767 & 0.750 \\
\hline $\mathrm{XFe}^{3+}$ & 0.602 & 0.636 & 0.609 & 0.544 & 0.501 & 0.791 & 0.796 & 0.738 & 0.759 & 0.747 & 0.793 & 0.904 & 0.985 & 0.790 \\
\hline
\end{tabular}


Table 3 Chemical compositions of epidote.

\begin{tabular}{|c|c|c|c|c|c|c|c|c|c|c|c|c|c|c|}
\hline Sample No. & YB108 & YB108 & YB120 & YB120 & ST2204 & ST2204 & YB154 & YB155 & YB159 & YB159 & YB35 & YB35 & YB46 & YB46 \\
\hline Rock type & BS & BS & QS & QS & BS & BS & QS & QS & QS & QS & BS & BS & BS & BS \\
\hline Grain No. & 7 & 7 & 2 & 2 & 7,1 & 7,1 & 16 & 25 & 34,1 & 34,2 & 2 & 2 & 11,1 & 16 \\
\hline \multirow[t]{2}{*}{ Point No. } & 33 & 36 & 21 & 16 & 53 & 56 & 60 & 10 & 137 & 136 & 37 & 41 & 13 & 80 \\
\hline & core & $\mathrm{rim}$ & & & core & rim & & & & & core & $\mathrm{rim}$ & & \\
\hline $\mathrm{SiO}_{2}$ & 38.4 & 37.5 & 37.3 & 37.6 & 37.2 & 38.2 & 37.2 & 37.1 & 37.5 & 37.3 & 37.2 & 37.6 & 37.1 & 38.1 \\
\hline $\mathrm{TiO}_{2}$ & 0.04 & 0.10 & 0.05 & 0.01 & 0.07 & 0.09 & 0.06 & 0.08 & 0.16 & 0.05 & 0.08 & 0.08 & 0.12 & 0.08 \\
\hline $\mathrm{Al}_{2} \mathrm{O}_{3}$ & 25.7 & 22.6 & 23.1 & 22.4 & 22.6 & 23.7 & 21.2 & 22.5 & 21.6 & 21.5 & 22.4 & 23.8 & 22.3 & 24.0 \\
\hline $\mathrm{Cr}_{2} \mathrm{O}_{3}$ & 0.02 & 0.00 & 0.00 & 0.00 & 0.00 & 0.00 & 0.07 & 0.03 & 0.00 & 0.05 & 0.02 & 0.02 & 0.00 & 0.01 \\
\hline $\mathrm{Fe}_{2} \mathrm{O}_{3}$ * & 10.4 & 13.6 & 13.1 & 14.1 & 15.2 & 13.3 & 16.0 & 12.8 & 14.6 & 14.8 & 15.0 & 13.2 & 15.0 & 12.7 \\
\hline $\mathrm{MnO}$ & 0.21 & 0.30 & 0.83 & 0.70 & 0.49 & 0.19 & 0.59 & 1.19 & 0.79 & 0.67 & 0.78 & 0.34 & 0.12 & 0.05 \\
\hline $\mathrm{MgO}$ & 0.02 & 0.01 & 0.06 & 0.03 & 0.02 & 0.07 & 0.01 & 0.07 & 0.03 & 0.00 & 0.00 & 0.09 & 0.02 & 0.00 \\
\hline $\mathrm{CaO}$ & 23.6 & 22.6 & 22.2 & 21.2 & 22.9 & 23.6 & 22.0 & 22.0 & 22.5 & 22.7 & 22.2 & 22.9 & 23.2 & 23.6 \\
\hline $\mathrm{Na}_{2} \mathrm{O}$ & 0.01 & 0.01 & 0.01 & 0.04 & 0.00 & 0.01 & 0.00 & 0.00 & 0.00 & 0.04 & 0.02 & 0.00 & 0.00 & 0.05 \\
\hline $\mathrm{K}_{2} \mathrm{O}$ & 0.00 & 0.00 & 0.00 & 0.00 & 0.00 & 0.00 & 0.00 & 0.00 & 0.03 & 0.00 & 0.00 & 0.00 & 0.01 & 0.00 \\
\hline \multirow[t]{2}{*}{ Total } & 98.4 & 96.7 & 96.7 & 96.1 & 98.5 & 99.2 & 97.1 & 95.8 & 97.2 & 97.1 & 97.7 & 98.0 & 97.9 & 98.6 \\
\hline & \multicolumn{3}{|c|}{ Atomic ratios $(O=12.5)$} & & & & & & & & & & & \\
\hline $\mathrm{Si}$ & 3.008 & 3.026 & 3.010 & 3.049 & 2.969 & 3.002 & 3.016 & 3.026 & 3.030 & 3.021 & 2.989 & 2.988 & 2.978 & 3.006 \\
\hline $\mathrm{Ti}$ & 0.002 & 0.006 & 0.003 & 0.001 & 0.004 & 0.005 & 0.004 & 0.005 & 0.010 & 0.003 & 0.005 & 0.005 & 0.007 & 0.005 \\
\hline $\mathrm{Al}$ & 2.373 & 2.149 & 2.197 & 2.141 & 2.126 & 2.195 & 2.025 & 2.163 & 2.057 & 2.052 & 2.122 & 2.229 & 2.110 & 2.232 \\
\hline $\mathrm{Cr}$ & 0.001 & 0.000 & 0.000 & 0.000 & 0.000 & 0.000 & 0.004 & 0.002 & 0.000 & 0.003 & 0.001 & 0.001 & 0.000 & 0.001 \\
\hline $\mathrm{Fe}^{3+}$ & 0.613 & 0.823 & 0.796 & 0.861 & 0.914 & 0.789 & 0.976 & 0.784 & 0.885 & 0.901 & 0.907 & 0.791 & 0.906 & 0.752 \\
\hline $\mathrm{Mn}$ & 0.014 & 0.021 & 0.057 & 0.048 & 0.033 & 0.013 & 0.041 & 0.082 & 0.054 & 0.046 & 0.053 & 0.023 & 0.008 & 0.003 \\
\hline $\mathrm{Mg}$ & 0.002 & 0.001 & 0.007 & 0.004 & 0.002 & 0.008 & 0.001 & 0.009 & 0.004 & 0.000 & 0.000 & 0.011 & 0.002 & 0.000 \\
\hline $\mathrm{Ca}$ & 1.981 & 1.954 & 1.919 & 1.842 & 1.958 & 1.987 & 1.911 & 1.923 & 1.948 & 1.970 & 1.911 & 1.950 & 1.995 & 1.995 \\
\hline $\mathrm{Na}$ & 0.002 & 0.002 & 0.002 & 0.006 & 0.000 & 0.002 & 0.000 & 0.000 & 0.000 & 0.006 & 0.003 & 0.000 & 0.000 & 0.008 \\
\hline $\mathrm{K}$ & 0.000 & 0.000 & 0.000 & 0.000 & 0.000 & 0.000 & 0.000 & 0.000 & 0.003 & 0.000 & 0.000 & 0.000 & 0.001 & 0.000 \\
\hline Total & 7.997 & 7.982 & 7.991 & 7.952 & 8.007 & 8.001 & 7.978 & 7.994 & 7.991 & 8.001 & 7.992 & 7.997 & 8.008 & 8.001 \\
\hline $\mathrm{YFe}^{3+}$ & 0.205 & 0.277 & 0.266 & 0.287 & 0.301 & 0.264 & 0.325 & 0.266 & 0.301 & 0.305 & 0.300 & 0.262 & 0.300 & 0.252 \\
\hline
\end{tabular}

Table 4 Chemical compositions of chlorite.

\begin{tabular}{|c|c|c|c|c|c|c|c|c|c|c|c|c|c|c|}
\hline Sample No. & YB108 & YB108 & YB120 & YB120 & ST2204 & ST2204 & ST2204 & YB154 & YB154 & YB155 & YB159 & YB35 & YB46 & YB46 \\
\hline Rock type & BS & BS & QS & QS & BS & BS & BS & QS & QS & QS & QS & BS & BS & BS \\
\hline Grain No. & 7,1 & 7,2 & 5 & 1 & 11 & 16 & 17 & 12 & 13 & 29 & 31 & 3,1 & 11 & 16 \\
\hline Point No. & 42 & 45 & 108 & 129 & 86 & 92 & 93 & 84 & 112 & 79 & 129 & 55 & 10 & 78 \\
\hline $\mathrm{SiO}_{2}$ & 26.2 & 26.4 & 27.4 & 27.4 & 26.6 & 26.5 & 26.3 & 26.2 & 26.9 & 28.0 & 26.6 & 25.9 & 27.2 & 27.8 \\
\hline $\mathrm{TiO}_{2}$ & 0.02 & 0.02 & 0.03 & 0.02 & 0.02 & 0.06 & 0.15 & 0.10 & 0.08 & 0.00 & 0.03 & 0.02 & 0.08 & 0.00 \\
\hline $\mathrm{Al}_{2} \mathrm{O}_{3}$ & 18.6 & 18.7 & 19.6 & 19.6 & 18.7 & 19.1 & 18.7 & 19.0 & 19.0 & 18.8 & 18.8 & 18.5 & 19.4 & 19.2 \\
\hline $\mathrm{Cr}_{2} \mathrm{O}_{3}$ & 0.00 & 0.00 & 0.03 & 0.04 & 0.04 & 0.04 & 0.00 & 0.00 & 0.00 & 0.01 & 0.05 & 0.02 & 0.00 & 0.00 \\
\hline $\mathrm{FeO}$ & 23.2 & 24.5 & 17.6 & 18.4 & 24.2 & 22.4 & 24.3 & 23.2 & 24.0 & 16.8 & 21.9 & 28.7 & 19.5 & 17.9 \\
\hline $\mathrm{MnO}$ & 0.45 & 0.45 & 0.77 & 0.99 & 0.45 & 0.42 & 0.58 & 0.41 & 0.51 & 0.99 & 1.04 & 0.40 & 0.26 & 0.26 \\
\hline $\mathrm{MgO}$ & 16.8 & 16.2 & 20.1 & 20.1 & 16.5 & 17.4 & 16.5 & 16.8 & 16.3 & 21.4 & 17.3 & 13.2 & 19.6 & 20.1 \\
\hline $\mathrm{CaO}$ & 0.04 & 0.03 & 0.03 & 0.00 & 0.09 & 0.07 & 0.08 & 0.02 & 0.01 & 0.04 & 0.04 & 0.03 & 0.01 & 0.02 \\
\hline $\mathrm{Na}_{2} \mathrm{O}$ & 0.00 & 0.00 & 0.00 & 0.01 & 0.00 & 0.00 & 0.02 & 0.00 & 0.00 & 0.03 & 0.03 & 0.00 & 0.00 & 0.00 \\
\hline $\mathrm{K}_{2} \mathrm{O}$ & 0.00 & 0.01 & 0.01 & 0.00 & 0.02 & 0.00 & 0.01 & 0.01 & 0.00 & 0.00 & 0.00 & 0.02 & 0.00 & 0.00 \\
\hline \multirow[t]{2}{*}{ Total } & 85.3 & 86.3 & 85.6 & 86.6 & 86.6 & 86.0 & 86.6 & 85.7 & 86.8 & 86.1 & 85.8 & 86.8 & 86.1 & 85.3 \\
\hline & \multicolumn{14}{|c|}{ Atomic ratios $(O=14)$} \\
\hline $\mathrm{Si}$ & 2.818 & 2.822 & 2.843 & 2.826 & 2.828 & 2.810 & 2.802 & 2.801 & 2.846 & 2.880 & 2.830 & 2.819 & 2.830 & 2.889 \\
\hline${ }^{[4]} \mathrm{Al}$ & 1.182 & 1.178 & 1.157 & 1.174 & 1.172 & 1.190 & 1.198 & 1.199 & 1.154 & 1.120 & 1.170 & 1.181 & 1.170 & 1.111 \\
\hline${ }^{[6]} \mathrm{Al}$ & 1.175 & 1.179 & 1.241 & 1.209 & 1.172 & 1.198 & 1.150 & 1.195 & 1.215 & 1.159 & 1.187 & 1.192 & 1.208 & 1.241 \\
\hline $\mathrm{Ti}$ & 0.002 & 0.002 & 0.002 & 0.002 & 0.002 & 0.005 & 0.012 & 0.008 & 0.006 & 0.000 & 0.002 & 0.002 & 0.006 & 0.000 \\
\hline $\mathrm{Cr}$ & 0.000 & 0.000 & 0.002 & 0.003 & 0.003 & 0.003 & 0.000 & 0.000 & 0.000 & 0.001 & 0.004 & 0.002 & 0.000 & 0.000 \\
\hline $\mathrm{Fe}^{2+}$ & 2.087 & 2.191 & 1.527 & 1.587 & 2.152 & 1.987 & 2.165 & 2.074 & 2.124 & 1.445 & 1.948 & 2.612 & 1.697 & 1.556 \\
\hline $\mathrm{Mn}$ & 0.041 & 0.041 & 0.068 & 0.086 & 0.041 & 0.038 & 0.052 & 0.037 & 0.046 & 0.086 & 0.094 & 0.037 & 0.023 & 0.023 \\
\hline $\mathrm{Mg}$ & 2.693 & 2.582 & 3.110 & 3.091 & 2.616 & 2.751 & 2.621 & 2.677 & 2.571 & 3.281 & 2.744 & 2.142 & 3.040 & 3.114 \\
\hline $\mathrm{Ca}$ & 0.005 & 0.003 & 0.003 & 0.000 & 0.010 & 0.008 & 0.009 & 0.002 & 0.001 & 0.004 & 0.005 & 0.003 & 0.001 & 0.002 \\
\hline $\mathrm{Na}$ & 0.000 & 0.000 & 0.000 & 0.002 & 0.000 & 0.000 & 0.004 & 0.000 & 0.000 & 0.006 & 0.006 & 0.000 & 0.000 & 0.000 \\
\hline $\mathrm{K}$ & 0.000 & 0.001 & 0.001 & 0.000 & 0.003 & 0.000 & 0.001 & 0.001 & 0.000 & 0.000 & 0.000 & 0.003 & 0.000 & 0.000 \\
\hline Total & 10.002 & 9.998 & 9.955 & 9.980 & 9.998 & 9.989 & 10.015 & 9.995 & 9.963 & 9.983 & 9.990 & 9.993 & 9.975 & 9.935 \\
\hline $\mathrm{XMg}$ & 0.563 & 0.541 & 0.671 & 0.661 & 0.549 & 0.581 & 0.548 & 0.563 & 0.548 & 0.694 & 0.585 & 0.451 & 0.642 & 0.667 \\
\hline
\end{tabular}


Table 5 Chemical compositions of garnet.

\begin{tabular}{|c|c|c|c|c|c|c|c|c|c|c|c|c|c|c|}
\hline Sample No. & YB120 & YB120 & YB120 & YB120 & YB120 & YB120 & YB120 & YB120 & YB154 & YB154 & YB154 & YB154 & YB154 & YB154 \\
\hline Rock type & QS & QS & QS & QS & QS & QS & QS & QS & QS & QS & QS & QS & QS & QS \\
\hline Grain No. & 7 & 7 & 4 & 4 & $x$ & $x$ & 2 & 2 & 14 & 14 & 14 & 14 & 14 & 14 \\
\hline \multirow[t]{2}{*}{ Point No. } & 5 & 2 & 146 & 142 & 161 & 153 & 166 & 164 & 13 & 2 & 58 & 53 & 64 & 60 \\
\hline & core & rim & core & rim & core & rim & core & rim & core & rim & core & rim & core & $\mathrm{rim}$ \\
\hline $\mathrm{SiO}_{2}$ & 36.5 & 37.3 & 36.4 & 37.3 & 36.7 & 37.0 & 36.9 & 37.3 & 37.4 & 37.4 & 37.3 & 37.6 & 37.7 & 37.1 \\
\hline $\mathrm{TiO}_{2}$ & 0.11 & 0.04 & 0.16 & 0.14 & 0.19 & 0.10 & 0.11 & 0.10 & 0.09 & 0.01 & 0.12 & 0.07 & 0.08 & 0.05 \\
\hline $\mathrm{Al}_{2} \mathrm{O}_{3}$ & 19.8 & 20.9 & 19.8 & 20.8 & 19.6 & 20.7 & 20.0 & 20.6 & 20.9 & 21.3 & 20.6 & 21.1 & 20.8 & 20.5 \\
\hline $\mathrm{Cr}_{2} \mathrm{O}_{3}$ & 0.00 & 0.04 & 0.00 & 0.01 & 0.00 & 0.00 & 0.00 & 0.01 & 0.03 & 0.02 & 0.01 & 0.01 & 0.05 & 0.01 \\
\hline $\mathrm{FeO}^{*}$ & 12.8 & 18.2 & 13.7 & 18.7 & 12.3 & 17.1 & 13.9 & 17.2 & 26.5 & 27.9 & 26.1 & 27.1 & 27.4 & 27.9 \\
\hline $\mathrm{MnO}$ & 22.9 & 15.0 & 22.8 & 14.7 & 24.4 & 17.0 & 24.6 & 16.5 & 8.06 & 5.96 & 7.94 & 6.45 & 6.57 & 5.51 \\
\hline $\mathrm{MgO}$ & 0.72 & 1.81 & 0.67 & 1.85 & 0.42 & 1.30 & 0.84 & 1.35 & 1.37 & 2.21 & 1.27 & 2.20 & 1.63 & 1.99 \\
\hline $\mathrm{CaO}$ & 5.64 & 6.08 & 5.52 & 6.63 & 6.42 & 6.67 & 4.43 & 6.98 & 5.95 & 5.83 & 6.90 & 6.05 & 6.61 & 6.18 \\
\hline Total & 98.5 & 99.4 & 99.1 & 100.1 & 100.0 & 99.9 & 100.8 & 100.0 & 100.3 & 100.6 & 100.2 & 100.6 & 100.8 & 99.2 \\
\hline $\mathrm{Fe}_{2} \mathrm{O}_{3}$ & 1.18 & 0.10 & 1.42 & 0.63 & 2.02 & 0.63 & 1.65 & 0.76 & 0.24 & 0.14 & 0.76 & 0.31 & 0.65 & 0.72 \\
\hline $\mathrm{FeO}$ & 11.7 & 18.1 & 12.4 & 18.1 & 10.5 & 16.5 & 12.4 & 16.5 & 26.3 & 27.8 & 25.4 & 26.8 & 26.8 & 27.3 \\
\hline \multirow[t]{2}{*}{ New total } & 98.6 & 99.4 & 99.2 & 100.2 & 100.3 & 99.9 & 100.9 & 100.1 & 100.3 & 100.7 & 100.3 & 100.6 & 100.9 & 99.4 \\
\hline & \multicolumn{14}{|c|}{ Atomic ratios $(O=12)$} \\
\hline $\mathrm{Si}$ & 3.004 & 3.007 & 2.986 & 2.988 & 2.985 & 2.985 & 2.984 & 2.998 & 3.004 & 2.983 & 2.997 & 2.996 & 3.004 & 3.000 \\
\hline $\mathrm{Ti}$ & 0.007 & 0.002 & 0.010 & 0.008 & 0.012 & 0.006 & 0.007 & 0.006 & 0.005 & 0.001 & 0.007 & 0.004 & 0.005 & 0.003 \\
\hline $\mathrm{Al}$ & 1.920 & 1.986 & 1.915 & 1.964 & 1.879 & 1.968 & 1.906 & 1.951 & 1.978 & 2.002 & 1.951 & 1.981 & 1.954 & 1.954 \\
\hline $\mathrm{Cr}$ & 0.000 & 0.003 & 0.000 & 0.001 & 0.000 & 0.000 & 0.000 & 0.001 & 0.002 & 0.001 & 0.001 & 0.001 & 0.003 & 0.001 \\
\hline $\mathrm{Fe}^{3+}$ & 0.073 & 0.006 & 0.088 & 0.038 & 0.124 & 0.038 & 0.100 & 0.046 & 0.015 & 0.009 & 0.046 & 0.019 & 0.039 & 0.044 \\
\hline $\mathrm{Fe}^{2+}$ & 0.808 & 1.221 & 0.852 & 1.215 & 0.713 & 1.115 & 0.840 & 1.110 & 1.765 & 1.852 & 1.708 & 1.787 & 1.787 & 1.843 \\
\hline $\mathrm{Mn}$ & 1.596 & 1.024 & 1.584 & 0.998 & 1.681 & 1.161 & 1.685 & 1.123 & 0.548 & 0.403 & 0.540 & 0.435 & 0.443 & 0.377 \\
\hline $\mathrm{Mg}$ & 0.088 & 0.218 & 0.082 & 0.221 & 0.051 & 0.156 & 0.101 & 0.162 & 0.164 & 0.263 & 0.152 & 0.261 & 0.194 & 0.240 \\
\hline $\mathrm{Ca}$ & 0.497 & 0.525 & 0.485 & 0.569 & 0.559 & 0.576 & 0.384 & 0.601 & 0.512 & 0.498 & 0.594 & 0.516 & 0.564 & 0.535 \\
\hline Total & 7.993 & 7.993 & 8.002 & 8.002 & 8.003 & 8.006 & 8.006 & 7.997 & 7.993 & 8.011 & 7.997 & 8.000 & 7.993 & 7.998 \\
\hline
\end{tabular}

Table 5 Chemical compositions of garnet (continued).

\begin{tabular}{|c|c|c|c|c|c|c|c|c|c|c|c|c|}
\hline Sample No & YB154 & YB154 & YB155 & YB155 & YB155 & YB155 & YB159 & YB159 & YB159 & YB159 & YB159 & YB159 \\
\hline Rock type & QS & QS & QS & QS & QS & QS & QS & QS & QS & QS & QS & QS \\
\hline Grain No. & 14 & 14 & 31 & 31 & 31 & 31 & 35 & 35 & 35 & 31 & 31 & 31 \\
\hline \multirow[t]{2}{*}{ Point No. } & 74 & 67 & 21 & 17 & 26 & 23 & 34 & 31 & 30 & 47 & 40 & 38 \\
\hline & core & rim & core & rim & core & rim & core & inter & rim & core & inter & rim \\
\hline $\mathrm{SiO}_{2}$ & 37.5 & 37.3 & 37.4 & 37.5 & 36.9 & 37.9 & 36.9 & 37.6 & 37.4 & 36.7 & 37.4 & 37.1 \\
\hline $\mathrm{TiO}_{2}$ & 0.10 & 0.05 & 0.08 & 0.07 & 0.09 & 0.06 & 0.18 & 0.07 & 0.05 & 0.11 & 0.05 & 0.04 \\
\hline $\mathrm{Al}_{2} \mathrm{O}_{3}$ & 20.8 & 21.0 & 21.0 & 21.0 & 20.6 & 21.0 & 20.3 & 20.8 & 20.9 & 20.5 & 20.6 & 20.8 \\
\hline $\mathrm{Cr}_{2} \mathrm{O}_{3}$ & 0.00 & 0.00 & 0.00 & 0.03 & 0.01 & 0.00 & 0.01 & 0.00 & 0.05 & 0.00 & 0.00 & 0.02 \\
\hline $\mathrm{FeO}^{*}$ & 26.1 & 27.2 & 12.7 & 14.1 & 12.3 & 13.6 & 9.16 & 23.8 & 26.9 & 7.60 & 21.9 & 26.7 \\
\hline $\mathrm{MnO}$ & 8.67 & 6.16 & 23.0 & 18.7 & 21.6 & 18.9 & 28.2 & 10.3 & 8.52 & 30.0 & 1.9 & 8.41 \\
\hline $\mathrm{MgO}$ & 1.17 & 2.58 & 2.31 & 2.71 & 2.06 & 2.70 & 0.81 & 1.35 & 2.01 & 0.65 & 1.31 & 2.04 \\
\hline $\mathrm{CaO}$ & 6.37 & 5.35 & 4.01 & 5.55 & 4.80 & 6.10 & 4.77 & 6.81 & 4.71 & 4.75 & 6.75 & 3.71 \\
\hline Total & 100.7 & 99.6 & 100.5 & 99.7 & 98.4 & 100.3 & 100.3 & 100.7 & 100.5 & 100.3 & 99.9 & 98.8 \\
\hline $\mathrm{Fe}_{2} \mathrm{O}_{3}$ & 0.51 & 0.22 & 0.38 & 0.2 & .20 & 0.43 & 1.05 & 0.62 & 0.43 & 0.84 & 0.62 & 0.00 \\
\hline $\mathrm{FeO}$ & 20.0 & 27.0 & 12.4 & a & .1 & 2 & 21 & 23.2 & 26.5 & 6.84 & 21.3 & 26.7 \\
\hline \multirow[t]{2}{*}{ New total } & 100.7 & 99.7 & 100.6 & 99.7 & 98.4 & 100.3 & 100.4 & 100.8 & 100.6 & 100.4 & 99.9 & 98.8 \\
\hline & \multicolumn{12}{|c|}{ Atomic ratios $(O=12)$} \\
\hline $\mathrm{Si}$ & 3.003 & 2.995 & 2.991 & 3.000 & 3.007 & 3.009 & 2.987 & 3.003 & 2.996 & 2.977 & 10 & 3.018 \\
\hline $\mathrm{Ti}$ & 0.006 & 0.003 & 0.005 & 4 & 0. & 0. & 0. & 0 & & & & 0.002 \\
\hline $\mathrm{Al}$ & 1.963 & 1.988 & 1.980 & 1.980 & 1.979 & 1.965 & 1.937 & & & & 1. & 1.994 \\
\hline $\mathrm{Cr}$ & 0.000 & 0.000 & 0.000 & 0.002 & 0.001 & 0.000 & 0.001 & 0.000 & 0.003 & 0.000 & 0.000 & 0.001 \\
\hline $\mathrm{Fe}^{3+}$ & 0.031 & 0.013 & 0.023 & 0.016 & 0.012 & 0.026 & 0.064 & 0.037 & 0.026 & 0.051 & 0.037 & 0.000 \\
\hline $\mathrm{Fe}^{2+}$ & 1.717 & 1.813 & 0.827 & 0.928 & 0.826 & 0.877 & 0.556 & 1.552 & 1.776 & 0.464 & 1.437 & 1.816 \\
\hline Mn & 0.588 & 0.419 & 1.558 & 1.267 & 1.491 & 1.271 & 1.934 & 0.697 & 0.578 & 2.061 & 0.811 & 0.579 \\
\hline $\mathrm{Mg}$ & 0.140 & 0.309 & 0.275 & 0.323 & 0.250 & 0.320 & 0.098 & 0.161 & 0.240 & 0.079 & 0.157 & 0.247 \\
\hline $\mathrm{Ca}$ & 0.547 & 0.460 & 0.344 & 0.476 & 0.419 & & & 0.583 & 0.404 & 0.413 & 0.582 & 0.323 \\
\hline Total & 7.994 & 8.001 & 8.003 & 7.996 & 7.991 & 7.991 & 8.001 & 7.995 & 8.000 & 8.011 & 7.991 & 7.982 \\
\hline
\end{tabular}


Table 6 Chemical compositions of muscovite.

\begin{tabular}{|c|c|c|c|c|c|c|c|c|c|c|c|c|c|c|}
\hline Sample No. & YB108 & YB108 & YB120 & YB120 & ST2204 & ST2204 & ST2204 & YB154 & YB155 & YB159 & YB159 & YB159 & YB35 & YB46 \\
\hline Rock type & BS & BS & QS & QS & BS & BS & BS & QS & QS & QS & QS & QS & BS & BS \\
\hline Grain No. & 8 & 8 & 6,1 & 6,2 & 17,1 & 17,2 & 11 & 17 & 25 & 20,1 & 20,2 & 25 & 2 & 12 \\
\hline Point No. & 46 & 47 & 262 & 270 & 95 & 100 & 104 & 57 & 11 & 64 & 65 & 91 & 34 & 17 \\
\hline $\mathrm{SiO}_{2}$ & 47.7 & 49.0 & 48.9 & 47.9 & 48.0 & 47.9 & 47.8 & 47.7 & 47.6 & 49.2 & 48.7 & 48.4 & 47.8 & 49.0 \\
\hline $\mathrm{TiO}_{2}$ & 0.52 & 0.36 & 0.39 & 0.37 & 0.49 & 0.62 & 0.56 & 0.37 & 0.46 & 0.26 & 0.47 & 0.30 & 0.53 & 0.57 \\
\hline $\mathrm{Al}_{2} \mathrm{O}_{3}$ & 27.2 & 26.8 & 26.8 & 28.6 & 26.6 & 26.6 & 27.1 & 27.2 & 28.0 & 27.3 & 26.6 & 26.8 & 27.1 & 26.2 \\
\hline $\mathrm{Cr}_{2} \mathrm{O}_{3}$ & 0.00 & 0.03 & 0.02 & 0.00 & 0.02 & 0.03 & 0.05 & 0.02 & 0.00 & 0.00 & 0.00 & 0.00 & 0.00 & 0.00 \\
\hline $\mathrm{FeO}^{\star}$ & 4.86 & 4.46 & 4.17 & 4.37 & 5.05 & 5.40 & 5.67 & 4.26 & 4.06 & 4.38 & 5.37 & 5.33 & 4.94 & 5.25 \\
\hline $\mathrm{MnO}$ & 0.00 & 0.04 & 0.00 & 0.01 & 0.04 & 0.02 & 0.03 & 0.08 & 0.01 & 0.02 & 0.00 & 0.01 & 0.00 & 0.02 \\
\hline $\mathrm{MgO}$ & 2.68 & 2.89 & 2.69 & 2.48 & 2.74 & 2.41 & 2.50 & 0.77 & 2.28 & 3.08 & 2.99 & 2.98 & 2.46 & 2.91 \\
\hline $\mathrm{CaO}$ & 0.02 & 0.00 & 0.02 & 0.03 & 0.05 & 0.01 & 0.02 & 0.02 & 0.00 & 0.02 & 0.02 & 0.02 & 0.01 & 0.01 \\
\hline $\mathrm{Na}_{2} \mathrm{O}$ & 0.76 & 0.70 & 1.03 & 1.12 & 0.50 & 0.59 & 0.60 & 0.88 & 1.16 & 0.76 & 0.52 & 0.74 & 1.00 & 0.52 \\
\hline $\mathrm{K}_{2} \mathrm{O}$ & 11.1 & 11.4 & 10.9 & 10.1 & 11.7 & 11.2 & 11.4 & 10.7 & 10.5 & 10.6 & 11.1 & 10.4 & 10.7 & 11.4 \\
\hline \multirow[t]{2}{*}{ Total } & 94.8 & 95.7 & 94.9 & 95.0 & 95.2 & 94.8 & 95.7 & 92.0 & 94.1 & 95.6 & 95.8 & 95.0 & 94.5 & 95.9 \\
\hline & \multicolumn{14}{|c|}{ Atomic ratios $(O=11)$} \\
\hline $\mathrm{Si}$ & 3.235 & 3.293 & 3.308 & 3.219 & 3.254 & 3.261 & 3.223 & 3.352 & 3.243 & 3.286 & 3.268 & 3.263 & 3.250 & 3.293 \\
\hline $\mathrm{Ti}$ & 0.027 & 0.018 & 0.020 & 0.019 & 0.025 & 0.032 & 0.028 & 0.020 & 0.024 & 0.013 & 0.024 & 0.015 & 0.027 & 0.029 \\
\hline $\mathrm{Al}$ & 2.174 & 2.122 & 2.137 & 2.265 & 2.126 & 2.135 & 2.153 & 2.253 & 2.248 & 2.149 & 2.104 & 2.130 & 2.172 & 2.075 \\
\hline $\mathrm{Cr}$ & 0.000 & 0.002 & 0.001 & 0.000 & 0.001 & 0.002 & 0.003 & 0.001 & 0.000 & 0.000 & 0.000 & 0.000 & 0.000 & 0.000 \\
\hline $\mathrm{Fe}^{3+}$ & 0.276 & 0.232 & 0.179 & 0.246 & 0.286 & 0.260 & 0.320 & 0.000 & 0.197 & 0.245 & 0.301 & 0.301 & 0.253 & 0.266 \\
\hline $\mathrm{Fe}^{2+}$ & 0.000 & 0.019 & 0.057 & 0.000 & 0.000 & 0.047 & 0.000 & 0.250 & 0.034 & 0.000 & 0.000 & 0.000 & 0.028 & 0.029 \\
\hline $\mathrm{Mn}$ & 0.000 & 0.002 & 0.000 & 0.001 & 0.002 & 0.001 & 0.002 & 0.005 & 0.001 & 0.001 & 0.000 & 0.001 & 0.000 & 0.001 \\
\hline $\mathrm{Mg}$ & 0.271 & 0.290 & 0.271 & 0.248 & 0.277 & 0.245 & 0.251 & 0.081 & 0.232 & 0.307 & 0.299 & 0.300 & 0.249 & 0.292 \\
\hline $\mathrm{Ca}$ & 0.001 & 0.000 & 0.001 & 0.002 & 0.004 & 0.001 & 0.001 & 0.002 & 0.000 & 0.001 & 0.001 & 0.001 & 0.001 & 0.001 \\
\hline $\mathrm{Na}$ & 0.100 & 0.091 & 0.135 & 0.146 & 0.066 & 0.078 & 0.078 & 0.120 & 0.153 & 0.098 & 0.068 & 0.097 & 0.132 & 0.068 \\
\hline $\mathrm{K}$ & 0.960 & 0.977 & 0.941 & 0.866 & 1.012 & 0.973 & 0.981 & 0.959 & 0.913 & 0.903 & 0.950 & 0.895 & 0.928 & 0.977 \\
\hline Total & 7.044 & 7.046 & 7.051 & 7.012 & 7.053 & 7.034 & 7.040 & 7.041 & 7.044 & 7.004 & 7.015 & 7.002 & 7.040 & 7.030 \\
\hline $\mathrm{XNa}$ & 0.094 & 0.085 & 0.126 & 0.144 & 0.061 & 0.074 & 0.074 & 0.111 & 0.144 & 0.098 & 0.066 & 0.098 & 0.124 & 0.065 \\
\hline
\end{tabular}

Table 7 Chemical compositions of albite.

\begin{tabular}{|c|c|c|c|c|c|c|c|c|c|c|}
\hline Sample No. & YB108 & YB120 & YB120 & ST2204 & YB154 & YB155 & YB159 & YB35 & YB35 & YB46 \\
\hline Rock type & BS & QS & QS & BS & QS & QS & QS & BS & BS & BS \\
\hline Grain No. & 5 & 8 & 10 & 15 & 23 & 29 & 25 & 4 & 3 & 18 \\
\hline Point No. & 40 & 98 & 142 & 84 & 1 & 78 & 86 & 1 & 54 & 101 \\
\hline $\mathrm{SiO}_{2}$ & 69.0 & 70.1 & 70.0 & 69.2 & 69.7 & 68.2 & 68.8 & 69.6 & 69.5 & 69.7 \\
\hline $\mathrm{TiO}_{2}$ & 0.00 & 0.01 & 0.00 & 0.00 & 0.00 & 0.00 & 0.00 & 0.00 & 0.02 & 0.00 \\
\hline $\mathrm{Al}_{2} \mathrm{O}_{3}$ & 19.4 & 19.6 & 19.7 & 19.5 & 19.5 & 19.1 & 19.8 & 19.7 & 19.6 & 20.0 \\
\hline $\mathrm{Cr}_{2} \mathrm{O}_{3}$ & 0.00 & 0.04 & 0.00 & 0.01 & 0.00 & 0.03 & 0.00 & 0.00 & 0.00 & 0.00 \\
\hline $\mathrm{FeO}$ & 0.05 & 0.00 & 0.00 & 0.07 & 0.10 & 0.07 & 0.15 & 0.04 & 0.10 & 0.03 \\
\hline $\mathrm{MnO}$ & 0.04 & 0.00 & 0.04 & 0.00 & 0.01 & 0.04 & 0.02 & 0.02 & 0.04 & 0.01 \\
\hline $\mathrm{MgO}$ & 0.00 & 0.01 & 0.00 & 0.01 & 0.00 & 0.00 & 0.00 & 0.00 & 0.01 & 0.00 \\
\hline $\mathrm{CaO}$ & 0.13 & 0.11 & 0.18 & 0.31 & 0.18 & 0.23 & 0.09 & 0.12 & 0.15 & 0.13 \\
\hline $\mathrm{Na}_{2} \mathrm{O}$ & 11.7 & 11.9 & 11.9 & 11.6 & 11.8 & 11.6 & 11.6 & 11.3 & 11.8 & 12.1 \\
\hline $\mathrm{K}_{2} \mathrm{O}$ & 0.04 & 0.03 & 0.01 & 0.04 & 0.03 & 0.01 & 0.03 & 0.06 & 0.06 & 0.02 \\
\hline \multirow[t]{2}{*}{ Total } & 100.4 & 101.8 & 101.8 & 100.7 & 101.3 & 99.3 & 100.5 & 100.8 & 101.3 & 102.0 \\
\hline & \multicolumn{10}{|c|}{ Atomic ratios $(O=8)$} \\
\hline $\mathrm{Si}$ & 3.002 & 3.005 & 3.001 & 3.000 & 3.004 & 3.001 & 2.990 & 3.006 & 2.998 & 2.987 \\
\hline $\mathrm{Ti}$ & 0.000 & 0.000 & 0.000 & 0.000 & 0.000 & 0.000 & 0.000 & 0.000 & 0.001 & 0.000 \\
\hline $\mathrm{Al}$ & 0.995 & 0.990 & 0.995 & 0.996 & 0.991 & 0.991 & 1.014 & 1.003 & 0.996 & 1.010 \\
\hline $\mathrm{Cr}$ & 0.000 & 0.001 & 0.000 & 0.000 & 0.000 & 0.001 & 0.000 & 0.000 & 0.000 & 0.000 \\
\hline $\mathrm{Fe}^{2+}$ & 0.002 & 0.000 & 0.000 & 0.003 & 0.004 & 0.003 & 0.005 & 0.001 & 0.004 & 0.001 \\
\hline $\mathrm{Mn}$ & 0.001 & 0.000 & 0.001 & 0.000 & 0.000 & 0.001 & 0.001 & 0.001 & 0.001 & 0.000 \\
\hline $\mathrm{Mg}$ & 0.000 & 0.001 & 0.000 & 0.001 & 0.000 & 0.000 & 0.000 & 0.000 & 0.001 & 0.000 \\
\hline $\mathrm{Ca}$ & 0.006 & 0.005 & 0.008 & 0.014 & 0.008 & 0.011 & 0.004 & 0.006 & 0.007 & 0.006 \\
\hline $\mathrm{Na}$ & 0.987 & 0.989 & 0.989 & 0.975 & 0.986 & 0.990 & 0.977 & 0.946 & 0.987 & 1.005 \\
\hline $\mathrm{K}$ & 0.002 & 0.002 & 0.001 & 0.002 & 0.002 & 0.001 & 0.002 & 0.003 & 0.003 & 0.001 \\
\hline Total & 4.995 & 4.994 & 4.996 & 4.991 & 4.995 & 4.998 & 4.993 & 4.967 & 4.998 & 5.011 \\
\hline $\mathrm{XCa}$ & 0.006 & 0.005 & 0.008 & 0.015 & 0.008 & 0.011 & 0.004 & 0.006 & 0.007 & 0.006 \\
\hline
\end{tabular}


Table 8 Chemical compositions of hematite.

\begin{tabular}{|c|c|c|c|c|c|c|c|c|}
\hline Sample No. & YB108 & YB108 & YB120 & ST2204 & ST2204 & ST2204 & ST2204 & ST2204 \\
\hline Rock type & BS & BS & QS & BS & BS & BS & BS & BS \\
\hline Grain No. & 5 & 4 & 4 & 7 & 7 & 7 & 8 & 8 \\
\hline Point No. & 30 & 52 & 25 & 74 & 77 & $65 \#$ & 72 & $71 \#$ \\
\hline $\mathrm{SiO}_{2}$ & 0.28 & 0.91 & 0.34 & 0.03 & 0.04 & 0.35 & 0.25 & 0.21 \\
\hline $\mathrm{TiO}_{2}$ & 0.03 & 0.08 & 4.74 & 7.62 & 9.01 & 2.28 & 9.38 & 2.72 \\
\hline $\mathrm{Al}_{2} \mathrm{O}_{3}$ & 0.27 & 0.91 & 0.23 & 0.05 & 0.00 & 0.07 & 0.07 & 0.01 \\
\hline $\mathrm{Cr}_{2} \mathrm{O}_{3}$ & 0.00 & 0.02 & 0.00 & 0.06 & 0.05 & 0.00 & .09 & 0.03 \\
\hline $\mathrm{FeO}^{*}$ & 89.1 & 86.4 & 85.3 & 84.2 & 82.5 & 87.3 & 81.9 & 87.4 \\
\hline $\mathrm{MnO}$ & 0.00 & 0.01 & 0.05 & 0.01 & 0.00 & .00 & .06 & 0.00 \\
\hline $\mathrm{MgO}$ & 0.13 & 0.34 & 0.02 & 0.00 & 0.01 & 0.02 & .06 & 0.06 \\
\hline $\mathrm{CaO}$ & 0.04 & 0.13 & 0.09 & 0.02 & 0.06 & 0.22 & 0.18 & 0.16 \\
\hline Total & 89.9 & 88.8 & 90.8 & 92.0 & 91.7 & 90.2 & 92.0 & 90.6 \\
\hline $\mathrm{Fe}_{2} \mathrm{O}_{3}$ & 98.9 & 95.6 & 89.8 & 86.0 & 82.7 & 94.6 & 81.8 & 94.5 \\
\hline $\mathrm{FeO}$ & 0.08 & .38 & 4.47 & & 8.06 & .15 & 3.34 & 2.39 \\
\hline \multirow[t]{2}{*}{ New total } & 99.7 & 98.4 & 99.7 & 100.6 & 090 & 99.7 & 100.2 & 100.1 \\
\hline & \multicolumn{7}{|c|}{ Atomic ratios $(\mathrm{O}=3)$} & \\
\hline $\mathrm{Si}$ & 0.007 & 0.024 & 0.009 & 0.001 & 0.001 & 0.009 & 0.007 & 0.006 \\
\hline $\mathrm{Ti}$ & 0.001 & 0.002 & 0.094 & 0.150 & 0.178 & 0.045 & 0.185 & 0.054 \\
\hline $\mathrm{Al}$ & 0.008 & 0.029 & 0.007 & 0.002 & 0.000 & 0.002 & 0.002 & 0.000 \\
\hline $\mathrm{Cr}$ & 0.000 & 0.000 & 0.000 & 0.001 & 0.001 & 0.000 & 0.002 & 0.001 \\
\hline $\mathrm{Fe}^{3+}$ & 1.975 & 1.919 & 1.786 & 1.695 & 1.640 & 1.888 & 1.613 & 1.880 \\
\hline $\mathrm{Fe}^{2+}$ & 0.002 & 0.008 & 0.099 & 0.150 & 0.177 & 0.048 & 0.183 & 0.053 \\
\hline $\mathrm{Mn}$ & 0.000 & 0.000 & 0.001 & 0.000 & 0.000 & 0.000 & 0.001 & 0.000 \\
\hline $\mathrm{Mg}$ & 0.005 & 0.014 & 0.001 & 0.000 & 0.000 & 0.001 & 0.002 & 0.002 \\
\hline $\mathrm{Ca}$ & 0.001 & 0.004 & 0.003 & 0.001 & 0.002 & 0.006 & 0.005 & 0.005 \\
\hline Total & 2.000 & 2.000 & 2.000 & 2.000 & 2.000 & 2.000 & 2.000 & 2.000 \\
\hline
\end{tabular}

Table 9 Chemical compositions of magnetite.

\begin{tabular}{|c|c|c|}
\hline \multicolumn{2}{|c|}{ Sample No. YB108 } & YB108 \\
\hline Rock type & BS & BS \\
\hline Grain No. & 5 & 5 \\
\hline Point No. & 27 & 28 \\
\hline$\overline{\mathrm{SiO}_{2}}$ & 0.67 & 0.62 \\
\hline $\mathrm{TiO}_{2}$ & 0.01 & 0.02 \\
\hline $\mathrm{Al}_{2} \mathrm{O}_{3}$ & 0.10 & 0.08 \\
\hline $\mathrm{Cr}_{2} \mathrm{O}_{3}$ & 0.00 & 0.03 \\
\hline $\mathrm{FeO}^{*}$ & 92.8 & 92.5 \\
\hline $\mathrm{MnO}$ & 0.00 & 0.01 \\
\hline $\mathrm{MgO}$ & 0.01 & 0.05 \\
\hline $\mathrm{CaO}$ & 0.04 & 0.02 \\
\hline Total & 93.6 & 93.3 \\
\hline $\mathrm{Fe}_{2} \mathrm{O}_{3}$ & 67.6 & 67.5 \\
\hline $\mathrm{FeO}$ & 32.0 & 31.8 \\
\hline New total & 100.4 & 100.1 \\
\hline \multicolumn{3}{|c|}{ Atomic ratios $(\mathrm{O}=4)$} \\
\hline $\mathrm{Si}$ & 0.026 & 0.024 \\
\hline $\mathrm{Ti}$ & 0.000 & 0.001 \\
\hline $\mathrm{Al}$ & 0.005 & 0.004 \\
\hline $\mathrm{Cr}$ & 0.000 & 0.001 \\
\hline $\mathrm{Fe}^{3+}$ & 1.944 & 1.947 \\
\hline $\mathrm{Fe}^{2+}$ & 1.024 & 1.020 \\
\hline $\mathrm{Mn}$ & 0.000 & 0.000 \\
\hline $\mathrm{Mg}$ & 0.001 & 0.003 \\
\hline $\mathrm{Ca}$ & 0.002 & 0.001 \\
\hline Total & 3.000 & 3.000 \\
\hline
\end{tabular}

Table 10 Chemical compositions of stilpnomelane.

\begin{tabular}{lcc}
\hline Sample No. & YB35 & YB35 \\
Rock type & $\mathrm{BS}$ & $\mathrm{BS}$ \\
Grain No. & 2 & 2 \\
Point No. & 35 & 36 \\
\hline $\mathrm{SiO}_{2}$ & 47.9 & 47.2 \\
$\mathrm{TiO}_{2}$ & 0.00 & 0.01 \\
$\mathrm{Al}_{2} \mathrm{O}_{3}$ & 6.01 & 6.01 \\
$\mathrm{Cr}_{2} \mathrm{O}_{3}$ & 0.04 & 0.00 \\
$\mathrm{FeO}$ & 27.1 & 26.9 \\
$\mathrm{MnO}$ & 1.25 & 1.39 \\
$\mathrm{MgO}$ & 7.28 & 7.01 \\
$\mathrm{CaO}$ & 0.52 & 0.56 \\
$\mathrm{Na} \mathrm{O}_{2} \mathrm{O}$ & 0.15 & 0.10 \\
$\mathrm{~K} 2$ & 0.52 & 0.51 \\
$\mathrm{Total}$ & 90.8 & 89.7 \\
$\mathrm{Atomic}$ & $\mathrm{ratios}(\mathrm{Si}=8)$ \\
$\mathrm{Si}$ & 8.000 & 8.000 \\
$\mathrm{Ti}$ & 0.000 & 0.001 \\
$\mathrm{Al}$ & 1.183 & 1.201 \\
$\mathrm{Cr}$ & 0.005 & 0.000 \\
$\mathrm{Fe}$ & 3.785 & 3.813 \\
$\mathrm{Mn}$ & 0.177 & 0.200 \\
$\mathrm{Mg}$ & 1.813 & 1.771 \\
$\mathrm{Ca}$ & 0.093 & 0.102 \\
$\mathrm{Na}$ & 0.049 & 0.033 \\
$\mathrm{~K}$ & 0.111 & 0.110 \\
$\mathrm{Total}$ & 15.215 & 15.230 \\
$\mathrm{XMg}$ & 0.324 & 0.317 \\
\hline \multicolumn{4}{l}{} \\
\hline
\end{tabular}

資料・解説

四国中央部三波川帯猿田川地域より産する含赤鉄鉱片岩中の角閃石の化学組成

\author{
坂野靖行
}

要旨

四国中央部三波川変成帯猿田川地域から産する含赤鉄鉱塩基性片岩及び石英片岩中の角閃石及び角閃石之共存 する鉱物の EPMA 分析を行い，角閃石 221 点及びその他の鉱物（緑れ九石・緑泥石・ざく万石・白雲母・曹長 石・赤鉄鉱・磁鉄鉱・スティルプノメレン) 90 点の分析值を示した. 分析が行われた試料は比較的高変成度である 曹長石一黒雲母帯から採集された。これらの化学組成デー夕は, 三波川変成帯猿田川地域における後退変成作用を 議論するために用いられた（Banno，2000）. 